\title{
ON STABLE LATTICES AND THE DIAGONAL GROUP
}

\author{
URI SHAPIRA AND BARAK WEISS
}

\begin{abstract}
Inspired by work of McMullen, we show that any orbit of the diagonal group in the space of lattices accumulates on the set of stable lattices. As consequences, we settle a conjecture of Ramharter concerning the asymptotic behavior of the Mordell constant, and reduce Minkowski's conjecture on products of linear forms to a geometric question, yielding two new proofs of the conjecture in dimensions up to 7 . We also answer a question of Harder on the volume of the set of stable lattices.
\end{abstract}

\section{INTRODUCTION}

Let $n \geq 2$ be an integer, let $G \stackrel{\text { def }}{=} \mathrm{SL}_{n}(\mathbb{R}), \Gamma \stackrel{\text { def }}{=} \mathrm{SL}_{n}(\mathbb{Z})$, let $A \subset G$ be the subgroup of positive diagonal matrices and let $\mathcal{L}_{n} \stackrel{\text { def }}{=} G / \Gamma$ be the space of unimodular lattices in $\mathbb{R}^{n}$. The purpose of this paper is to present a dynamical result regarding the action of $A$ on $\mathcal{L}_{n}$, and to present some consequences in the geometry of numbers.

A lattice $x \in \mathcal{L}_{n}$ is called stable if for any subgroup $\Lambda \subset x$, the covolume of $\Lambda$ in $\operatorname{span}(\Lambda)$ is at least 1 . In particular the length of the shortest nonzero vector in $x$ is at least 1 . Stable lattices have also been called 'semistable', they were introduced in a broad algebro-geometric context by Harder, Narasimhan and Stuhler [Stu76, HN74], and were used to develop a reduction theory for the study of the topology of locally symmetric spaces. See Grayson [Gra84] for a clear exposition.

Theorem 1.1. For any $x \in \mathcal{L}_{n}$, the orbit-closure $\overline{A x}$ contains a stable lattice.

Theorem 1.1 is inspired by a breakthrough result of McMullen [McM05]. Recall that a lattice in $\mathcal{L}_{n}$ is called well-rounded if its shortest nonzero vectors span $\mathbb{R}^{n}$. In connection with his work on Minkowski's conjecture, McMullen showed that the closure of any bounded $A$-orbit in $\mathcal{L}_{n}$ contains a well-rounded lattice. The set of well-rounded lattices neither contains, nor is contained in, the set of stable lattices, but the proof of Theorem 1.1 closely follows McMullen's strategy. 
We apply Theorem 1.1 to two problems in the geometry of numbers. Let $x \in \mathcal{L}_{n}$ be a unimodular lattice. By a symmetric box in $\mathbb{R}^{n}$ we mean a set of the form $\left[-a_{1}, a_{1}\right] \times \cdots \times\left[-a_{n}, a_{n}\right]$, and we say that a symmetric box is admissible for $x$ if it contains no nonzero points of $x$ in its interior. The Mordell constant of $x$ is defined to be

$$
\kappa(x) \stackrel{\text { def }}{=} \frac{1}{2^{n}} \sup _{\mathcal{B}} \operatorname{Vol}(\mathcal{B})
$$

where the supremum is taken over admissible symmetric boxes $\mathcal{B}$, and where $\operatorname{Vol}(\mathcal{B})$ denotes the volume of $\mathcal{B}$. We also write

$$
\kappa_{n} \stackrel{\text { def }}{=} \inf \left\{\kappa(x): x \in \mathcal{L}_{n}\right\} .
$$

The infimum in this definition is in fact a minimum, and, as with many problems in the geometry of numbers it is of interest to compute the constants $\kappa_{n}$ and identify the lattices realizing the minimum. However this appears to be a very difficult problem, which so far has only been solved for $n=2,3$, the latter in a difficult paper of Ramharter [Ram96]. It is also of interest to provide bounds on the asymptotics of $\kappa_{n}$, and in [Ram00], Ramharter conjectured that $\limsup _{n \rightarrow \infty} \kappa_{n}^{1 / n \log n}>0$. As a simple corollary of Theorem 1.1, we validate Ramharter's conjecture, with an explicit bound:

Corollary 1.2. For all $n \geq 2$,

$$
\kappa_{n} \geq n^{-n / 2} \text {. }
$$

In particular

$$
\kappa_{n}^{1 / n \log n} \geq n^{-1 / 2 \log n} \longrightarrow_{n \rightarrow \infty} \frac{1}{\sqrt{e}} .
$$

We remark that Corollary 1.2 could also be derived from McMullen's results and a theorem of Birch and Swinnerton-Dyer. In $\S 4$ we show that the bound (1.3) is not optimal and explain how to obtain better bounds, for all $n$ which are not divisible by 4 . We refer the reader to [SW] for more information on the possible values of $\kappa(x), x \in \mathcal{L}_{n}$.

Our second application concerns Minkowski's conjecture ${ }^{1}$, which posits that for any unimodular lattice $x$, one has

$$
\sup _{u \in \mathbb{R}^{n}} \inf _{v \in x}|N(u-v)| \leq \frac{1}{2^{n}}
$$

where $N\left(u_{1}, \ldots, u_{d}\right) \stackrel{\text { def }}{=} \prod_{j} u_{j}$. Minkowski solved the question for $n=2$ and several authors resolved the cases $n \leq 5$. In [McM05], McMullen settled the case $n=6$. In fact, using his theorem on the $A$-action

\footnotetext{
${ }^{1}$ It is not clear to us whether Minkowski actually made this conjecture.
} 
on $\mathcal{L}_{n}$, McMullen showed that in arbitrary dimension $n$, Minkowski's conjecture is implied by the statement that any well-rounded lattice $x \subset \mathbb{R}^{d}$ with $d \leq n$ satisfies

$$
\operatorname{covrad}(x) \leq \frac{\sqrt{d}}{2}
$$

where $\operatorname{covrad}(x) \stackrel{\text { def }}{=} \max _{u \in \mathbb{R}^{d}} \min _{v \in x}\|u-v\|$ and $\|\cdot\|$ is the Euclidean norm on $\mathbb{R}^{d}$. At the time of writing [McM05], (1.5) was known to hold for well-rounded lattices in dimension at most 6 , and in recent work of Hans-Gill, Raka, Sehmi and Leetika [HGRS09,HGRS11, RL], (1.5) has been proved for well-rounded lattices in dimensions $n=7,8,9$, thus settling Minkowski's question in those cases.

Our work gives two new approaches to Minkowski's conjecture. A direct application of Theorem 1.1 (see Corollary 5.1) shows that it follows in dimension $n$, from the assertion that for any stable $x \in \mathcal{L}_{n}$, (1.5) holds. Note that we do not require (1.5) in dimensions less than $n$. Using the strategy of Woods and Hans-Gill et al, in Theorem 5.8 we define a compact subset $\mathrm{KZS} \subset \mathbb{R}^{n}$ and a collection of $2^{n-1}$ subsets $\{\mathcal{W}(\mathcal{I})\}$ of $\mathbb{R}^{n}$. We show that the assertion $\mathrm{KZS} \subset \bigcup_{\mathcal{I}} \mathcal{W}(\mathcal{I})$ implies Minkowski's conjecture in dimension $n$.

Secondly, an induction using the naturality of stable lattices, leads to the following sufficient condition:

Corollary 1.3. Suppose that for some dimension $n$, for all $d \leq n$, any stable lattice $x \in \mathcal{L}_{d}$ which is a local maximum of the function covrad, satisfies (1.5). Then (1.4) holds for any $x \in \mathcal{L}_{n}$.

The local maxima of the function covrad have been studied in depth in recent work of Dutour-Sikirić, Schürmann and Vallentin [DSSV12], who characterized them and showed that there are finitely many in each dimension. These two approaches give two new proofs of Minkowski's Conjecture in dimensions $n \leq 7$.

A natural question is to what extent stable lattices are typical in $\mathcal{L}_{n}$. The definition of stability may appear at first sight to be very restrictive. Nevertheless in $\S 6$ we show that as $n \rightarrow \infty$, the probability that a random lattice is stable tends to 1 (where the probability is taken with respect to the natural $G$-invariant measure on $\mathcal{L}_{n}$ ). This answers a question of $\mathrm{G}$. Harder. In fact a stronger statement is true, see Proposition 6.2. For the results of $\S 6$ we use Siegel's approach to measure volumes [Sie45] and rely on computations of Thunder [Thu98].

A significant difference between Theorem 1.1 and McMullen's work on well-rounded lattices, is that we do not need to assume that the orbit $A x$ is bounded. One may wonder whether McMullen's result is 
valid without the hypothesis that $A x$ is bounded, namely is it true that the closure of any $A$-orbit in $\mathcal{L}_{n}$ (or even the orbit itself) contains a well-rounded lattice? We answer these questions affirmatively for closed orbits in $\S 7$. To this end we use results of Tomanov and the second-named author [TW03], as well as a covering result (which we learned from Michael Levin) generalizing one of the results of [McM05]; this topological result appears to be well-known to experts, but in order to keep this paper self-contained, we give the proof in the appendix. For another perspective on this and related questions, see [PS].

1.1. Acknowledgements. Our work was inspired by Curt McMullen's breakthrough paper [McM05] and many of our arguments are adaptations of arguments appearing in [McM05]. We are also grateful to Curt McMullen for additional insightful remarks, and in particular for the suggestion to study the set of stable lattices in connection with the $A$-action on $\mathcal{L}_{n}$. We thank Michael Levin for useful discussions on topological questions and for agreeing to include the proof of Theorem 7.2 in the appendix. We also thank Mathieu Dutour-Sikiric, Rajinder Hans-Gill, Günter Harder, Gregory Minton and Gerhard Ramharter for useful discussions. The authors' work was supported by ERC starter grant DLGAPS 279893 and ISF grant 190/08.

\section{Orbit Closures and stable lattices}

Given a lattice $x \in \mathcal{L}_{n}$ and a subgroup $\Lambda \subset x$, we denote by $r(\Lambda)$ the rank of $\Lambda$ and by $|\Lambda|$ the covolume of $\Lambda$ in the linear subspace $\operatorname{span}(\Lambda)$. Let

$$
\begin{aligned}
& \mathcal{V}(x) \stackrel{\text { def }}{=}\left\{|\Lambda|^{\frac{1}{r(\Lambda)}}: \Lambda \subset x\right\}, \\
& \alpha(x) \stackrel{\text { def }}{=} \min \mathcal{V}(x) .
\end{aligned}
$$

Since we may take $\Lambda=x$ we have $\alpha(x) \leq 1$ for all $x \in \mathcal{L}_{n}$, and $x$ is stable precisely if $\alpha(x)=1$. Observe that $\mathcal{V}(x)$ is a countable discrete subset of the positive reals, and hence the minimum in (2.1) is attained. Also note that the function $\alpha$ is a variant of the 'length of the shortest vector'; it is continuous and the sets $\{x: \alpha(x) \geq \varepsilon\}$ are an exhaustion of $\mathcal{L}_{n}$ by compact sets.

We begin by explaining the strategy for proving Theorem 1.1, which is identical to the one used by McMullen. For a lattice $x \in X$ and $\varepsilon>0$ we define an open cover $\mathcal{U}^{x, \varepsilon}=\left\{U_{k}^{x, \varepsilon}\right\}_{k=1}^{n}$ of the diagonal group $A$, where if $a \in U_{k}^{x, \varepsilon}$ then $\alpha(a x)$ is 'almost attained' by a subgroup of rank $k$. In particular, if $a \in U_{n}^{x, \varepsilon}$ then $a x$ is 'almost stable'. The main point is to show that for any $\varepsilon>0, U_{n}^{x, \varepsilon} \neq \varnothing$; for then, taking $\varepsilon_{j} \rightarrow 0$ 
and $a_{j} \in A$ such that $a_{j} \in U_{n}^{x, \varepsilon_{j}}$, we find (passing to a subsequence) that $a_{j} x$ converges to a stable lattice.

In order to establish that $U_{n}^{x, \varepsilon} \neq \varnothing$, we apply a topological result of McMullen (Theorem 3.3) regarding open covers which is reminiscent of the classical result of Lebesgue that asserts that in an open cover of Euclidean $n$-space by bounded balls there must be a point which is covered $n+1$ times. We will work to show that the cover $\mathcal{U}^{x, \varepsilon}$ satisfies the assumptions of Theorem 3.3. We will be able to verify these assumptions when the orbit $A x$ is bounded. In $\S 2.1$ we reduce the proof of Theorem 1.1 to this case.

2.1. Reduction to bounded orbits. Using a result of Birch and Swinnerton-Dyer, we will now show that it suffices to prove Theorem 1.1 under the assumption that the orbit $A x \subset \mathcal{L}_{n}$ is bounded; that is, that $\overline{A x}$ is compact. In this subsection we will denote $A, G$ by $A_{n}, G_{n}$ as various dimensions will appear.

For a matrix $g \in G_{n}$ we denote by $[g] \in \mathcal{L}_{n}$ the corresponding lattice. If

$$
g=\left(\begin{array}{cccc}
g_{1} & * & \ldots & * \\
0 & g_{2} & \ldots & \vdots \\
\vdots & & \ddots & * \\
0 & \ldots & 0 & g_{k}
\end{array}\right)
$$

where $g_{i} \in G_{n_{i}}$ for each $i$, then we say that $g$ is in upper triangular block form and refer to the $g_{i}$ 's as the diagonal blocks. Note that in this definition, we insist that each $g_{i}$ is of determinant one.

Lemma 2.1. Let $x=[g] \in \mathcal{L}_{n}$ where $g$ is in upper triangular block form as in (2.2) and for each $1 \leq i \leq k,\left[g_{i}\right]$ is a stable lattice in $\mathcal{L}_{n_{i}}$. Then $x$ is stable.

Proof. By induction, in proving the Lemma we may assume that $k=$ 2. Let us denote the standard basis of $\mathbb{R}^{n}$ by $\mathbf{e}_{1}, \ldots, \mathbf{e}_{n}$, let us write $n=n_{1}+n_{2}, V_{1} \stackrel{\text { def }}{=} \operatorname{span}\left\{\mathbf{e}_{1}, \ldots, \mathbf{e}_{n_{1}}\right\}, V_{2} \stackrel{\text { def }}{=} \operatorname{span}\left\{\mathbf{e}_{n_{1}+1} \ldots, \mathbf{e}_{n}\right\}$, and let $\pi: \mathbb{R}^{n} \rightarrow V_{2}$ be the natural projection. By construction we have $x \cap V_{1}=\left[g_{1}\right], \pi(x)=\left[g_{2}\right]$.

Let $\Lambda \subset x$ be a subgroup, write $\Lambda_{1} \stackrel{\text { def }}{=} \Lambda \cap V_{1}$ and choose a direct complement $\Lambda_{2} \subset \Lambda$, that is

$$
\Lambda=\Lambda_{1}+\Lambda_{2}, \quad \Lambda_{1} \cap \Lambda_{2}=\{0\} .
$$

We claim that

$$
|\Lambda|=\left|\Lambda_{1}\right| \cdot\left|\pi\left(\Lambda_{2}\right)\right| .
$$


To see this we recall that one may compute $|\Lambda|$ via the Gram-Schmidt process. Namely, one begins with a set of generators $v_{j}$ of $\Lambda$ and successively defines $u_{1}=v_{1}$ and $u_{j}$ is the orthogonal projection of $v_{j}$ on $\operatorname{span}\left(v_{1}, \ldots, v_{j-1}\right)^{\perp}$. In these terms, $|\Lambda|=\prod_{j}\left\|u_{j}\right\|$. Since $\pi$ is an orthogonal projection and $\Lambda \cap V_{1}$ is in $\operatorname{ker} \pi$, (2.3) is clear from the above description.

The discrete subgroup $\Lambda_{1}$, when viewed as a subgroup of $\left[g_{1}\right] \in \mathcal{L}_{n_{1}}$ satisfies $\left|\Lambda_{1}\right| \geq 1$ because $\left[g_{1}\right]$ is assumed to be stable. Similarly $\pi\left(\Lambda_{2}\right) \subset\left[g_{2}\right] \in \mathcal{L}_{n_{2}}$ satisfies $\left|\pi\left(\Lambda_{2}\right)\right| \geq 1$, hence $|\Lambda| \geq 1$.

Lemma 2.2. Let $x \in \mathcal{L}_{n}$ and assume that $\overline{A x}$ contains a lattice $[g]$ with $g$ of upper triangular block form as in (2.2). For each $1 \leq i \leq k$, suppose $\left[h_{i}\right] \in \overline{A_{n_{i}}\left[g_{i}\right]} \subset \mathcal{L}_{n_{i}}$. Then there exists a lattice $[h] \in \overline{A x}$ such that $h$ has the form (2.2) with $h_{i}$ as its diagonal blocks.

Proof. Let $\Omega$ be the set of all lattices $[g]$ of a fixed triangular form as in (2.2). Then $\Omega$ is a closed subset of $\mathcal{L}_{n}$ and there is a projection

$$
\tau: \Omega \rightarrow \mathcal{L}_{n_{1}} \times \cdots \times \mathcal{L}_{n_{k}}, \quad \tau([g])=\left(\left[g_{1}\right], \ldots,\left[g_{k}\right]\right) .
$$

The map $\tau$ has a compact fiber and is equivariant with respect to the action of $\widetilde{A} \stackrel{\text { def }}{=} A_{n_{1}} \times \cdots \times A_{n_{k}}$. By assumption, there is a sequence $\tilde{a}_{j}=\left(a_{1}^{(j)}, \ldots, a_{k}^{(j)}\right), a_{i}^{(j)} \in A_{n_{i}}$ in $\widetilde{A}$ such that $a_{i}^{(j)}\left[g_{i}\right] \rightarrow\left[h_{i}\right]$, then after passing to a subsequence, $\tilde{a}_{j}[g] \rightarrow[h]$ where $h$ has the required properties. Since $\overline{A x} \supset \overline{A[g]}$, the claim follows.

Lemma 2.3. Let $x \in \mathcal{L}_{n}$. Then there is $[g] \in \overline{A x}$ such that, up to a possible permutation of the coordinates, $g$ is of upper triangular block form as in (2.2) and each $A_{n_{i}}\left[g_{i}\right] \subset \mathcal{L}_{n_{i}}$ is bounded.

Proof. If the orbit $A x$ is bounded there is nothing to prove. According to Birch and Swinnerton-Dyer [BSD56], if $A x$ is unbounded then $\overline{A x}$ contains a lattice with a representative as in (2.2) (up to a possible permutation of the coordinates) with $k=2$. Now the claim follows using induction and appealing to Lemma 2.2.

Proposition 2.4. It is enough to establish Theorem 1.1 for lattices having a bounded $A$-orbit.

Proof. Let $x \in \mathcal{L}_{n}$ be arbitrary. By Lemma $2.3, \overline{A x}$ contains a lattice $[g]$ with $g$ of upper triangular block form (up to a possible permutation of the coordinates) with diagonal blocks representing lattices with bounded orbits under the corresponding diagonal groups. Assuming Theorem 1.1 for lattices having bounded orbits, and applying Lemma 2.2 we may take $g$ whose diagonal blocks represent stable lattices. By Lemma 2.1, $[g]$ is stable as well. 
2.2. Some technical preparations. We now discuss the subgroups of a lattice $x \in \mathcal{L}_{n}$ which almost attain the minimum $\alpha(x)$ in (2.1).

Definition 2.5. Given a lattice $x \in \mathcal{L}_{n}$ and $\delta>0$, let

$$
\begin{aligned}
\operatorname{Min}_{\delta}(x) & \stackrel{\text { def }}{=}\left\{\Lambda \subset x:|\Lambda|^{\frac{1}{r(\Lambda)}}<(1+\delta) \alpha(x)\right\}, \\
\mathbf{V}_{\delta}(x) & \stackrel{\text { def }}{=} \operatorname{span} \operatorname{Min}_{\delta}(x), \\
\operatorname{dim}_{\delta}(x) & \stackrel{\text { def }}{=} \operatorname{dim} \mathbf{V}_{\delta}(x) .
\end{aligned}
$$

We will need the following technical statement.

Lemma 2.6. For any $\rho>0$ there exists a neighborhood of the identity $W \subset G$ with the following property. Suppose $2 \rho \leq \delta_{0} \leq d+1$ and suppose $x \in \mathcal{L}_{n}$ such that $\operatorname{dim}_{\delta_{0}-\rho}(x)=\operatorname{dim}_{\delta_{0}+\rho}(x)$. Then for any $g \in W$ and any $\delta \in\left(\delta_{0}-\frac{\rho}{2}, \delta_{0}+\frac{\rho}{2}\right)$ we have

$$
\boldsymbol{V}_{\delta}(g x)=g \boldsymbol{V}_{\delta_{0}}(x) .
$$

In particular, there is $1 \leq k \leq n$ such that for any $g \in W$ and any $\delta \in\left(\delta_{0}-\frac{\rho}{2}, \delta_{0}+\frac{\rho}{2}\right), \operatorname{dim}_{\delta}(g x)=k$.

Proof. Let $c>1$ be chosen close enough to 1 so that for $2 \rho \leq \delta_{0} \leq d+1$ we have

$$
c^{2}\left(1+\delta_{0}+\frac{\rho}{2}\right)<1+\delta_{0}+\rho \text { and } \frac{1+\delta_{0}-\frac{\rho}{2}}{c^{2}}>1+\delta_{0}-\rho .
$$

Let $W$ be a small enough neighborhood of the identity in $G$, so that for any discrete subgroup $\Lambda \subset \mathbb{R}^{n}$ we have

$$
g \in W \quad \Longrightarrow \quad c^{-1}|\Lambda|^{\frac{1}{r(\Lambda)}} \leq|g \Lambda|^{\frac{1}{r(g \Lambda)}} \leq c|\Lambda|^{\frac{1}{r(\Lambda)}} .
$$

Such a neighborhood exists since the linear action of $G$ on $\bigoplus_{k=1}^{n} \bigwedge_{1}^{k} \mathbb{R}^{n}$ is continuous, and since we can write $|\Lambda|=\left\|v_{1} \wedge \cdots \wedge v_{r}\right\|$ where $v_{1}, \ldots, v_{r}$ is a generating set for $\Lambda$. It follows from (2.6) that for any $x \in \mathcal{L}_{n}$ and $g \in W$ we have

$$
c^{-1} \alpha(x) \leq \alpha(g x) \leq c \alpha(x) .
$$

Let $\delta \in\left(\delta_{0}-\frac{\rho}{2}, \delta_{0}+\frac{\rho}{2}\right)$ and $g \in W$. We will show below that

$$
g \operatorname{Min}_{\delta_{0}-\rho}(x) \subset \operatorname{Min}_{\delta}(g x) \subset g \operatorname{Min}_{\delta_{0}+\rho}(x) .
$$

Note first that (2.8) implies the assertion of the Lemma; indeed, since $\mathbf{V}_{\delta_{1}}(x) \subset \mathbf{V}_{\delta_{2}}(x)$ for $\delta_{1}<\delta_{2}$, and since we assumed that $\operatorname{dim}_{\delta_{0}-\rho}(x)=$ $\operatorname{dim}_{\delta_{0}+\rho}(x)$, we see that $\mathbf{V}_{\delta_{0}}(x)=\mathbf{V}_{\delta}(x)$ for $\delta_{0}-\rho \leq \delta \leq \delta_{0}+\rho$. So by (2.5), the subspaces spanned by the two sides of (2.8) are equal to $g \mathbf{V}_{\delta_{0}}(x)$ and (2.4) follows. 
It remains to prove (2.8). Let $\Lambda \in \operatorname{Min}_{\delta_{0}-\rho}(x)$. Then we find

$$
\begin{aligned}
|g \Lambda|^{\frac{1}{r(g \Lambda)}} & \stackrel{(2.6)}{\leq} c|\Lambda|^{\frac{1}{r(\Lambda)}} \leq c\left(1+\delta_{0}-\rho\right) \alpha(x) \\
& \quad \stackrel{(2.5)}{\leq} c^{-1}\left(1+\delta_{0}-\frac{\rho}{2}\right) \alpha(x) \stackrel{(2.7)}{<}(1+\delta) \alpha(g x) .
\end{aligned}
$$

By definition this means that $g \Lambda \in \operatorname{Min}_{\delta}(g x)$ which establishes the first inclusion in (2.8). The second inclusion is similar and is left to the reader.

2.3. The cover of $A$. Let $x \in \mathcal{L}_{n}$ and let $\varepsilon>0$ be given. Define $\mathcal{U}^{x, \varepsilon}=\left\{U_{i}^{x, \varepsilon}\right\}_{i=1}^{n}$ where

$$
U_{k}^{x, \varepsilon} \stackrel{\text { def }}{=}\left\{a \in A: \operatorname{dim}_{\delta}(a x)=k \text { for } \delta \text { in a neighborhood of } k \varepsilon\right\} \text {. }
$$

Theorem 2.7. Let $x \in \mathcal{L}_{n}$ be such that $A x$ is bounded. Then for any $\varepsilon \in(0,1), U_{n}^{x, \varepsilon} \neq \varnothing$.

In this subsection we will reduce the proof of Theorem 1.1 to Theorem 2.7. This will be done via the following statement, which could be interpreted as saying that a lattice satisfying $\operatorname{dim}_{\delta}(x)=n$ is 'almost stable'.

Lemma 2.8. For each $n$, there exists a positive function $\psi(\delta)$ with $\psi(\delta) \rightarrow_{\delta \rightarrow 0} 0$, such that for any $x \in \mathcal{L}_{n}$,

$$
\left\{\Lambda_{i}\right\}_{i=1}^{\ell} \subset \operatorname{Min}_{\delta}(x) \Longrightarrow \Lambda_{1}+\cdots+\Lambda_{\ell} \in \operatorname{Min}_{\psi(\delta)}(x)
$$

In particular, if $\operatorname{dim}_{\delta}(x)=n$ then $\alpha(x) \geq(1+\psi(\delta))^{-1}$.

Proof. Let $\Lambda, \Lambda^{\prime}$ be two discrete subgroups of $\mathbb{R}^{d}$. The following inequality is straightforward to prove via the Gram-Schmidt procedure for computing $|\Lambda|$ :

$$
\left|\Lambda+\Lambda^{\prime}\right| \leq \frac{|\Lambda| \cdot\left|\Lambda^{\prime}\right|}{\left|\Lambda \cap \Lambda^{\prime}\right|}
$$

Here we adopt the convention that $\left|\Lambda \cap \Lambda^{\prime}\right|=1$ when $\Lambda \cap \Lambda^{\prime}=\{0\}$. Let $x \in \mathcal{L}_{n}$ and let $\left\{\Lambda_{i}\right\}_{i=1}^{\ell} \subset \operatorname{Min}_{\delta}(x)$. Assume first that $\ell \leq n$. We prove by induction on $\ell$ the existence of a function $\psi_{\ell}(\delta) \stackrel{\delta \rightarrow 0}{\longrightarrow} 0$ for which $\Lambda_{1}+\cdots+\Lambda_{\ell} \in \operatorname{Min}_{\psi_{\ell}(\delta)}(x)$. For $\ell=1$ one can trivially pick $\psi_{1}(\delta)=\delta$. Assuming the existence of $\psi_{\ell-1}$, set $\Lambda=\Lambda_{1}, \Lambda^{\prime}=\Lambda_{2}+\cdots+\Lambda_{\ell}$, $\alpha=\alpha(x)$ and note that $r\left(\Lambda+\Lambda^{\prime}\right)=r(\Lambda)+r\left(\Lambda^{\prime}\right)-r\left(\Lambda \cap \Lambda^{\prime}\right)$. We deduce from (2.11) and the definitions that

$$
\begin{aligned}
\left|\Lambda+\Lambda^{\prime}\right| & \leq \frac{|\Lambda| \cdot\left|\Lambda^{\prime}\right|}{\left|\Lambda \cap \Lambda^{\prime}\right|} \leq \frac{((1+\delta) \alpha)^{r(\Lambda)}\left(\left(1+\psi_{\ell-1}(\delta)\right) \alpha\right)^{r\left(\Lambda^{\prime}\right)}}{\alpha^{r\left(\Lambda \cap \Lambda^{\prime}\right)}} \\
& =(1+\delta)^{r(\Lambda)}\left(1+\psi_{\ell-1}(\delta)\right)^{r\left(\Lambda^{\prime}\right)} \alpha^{r\left(\Lambda+\Lambda^{\prime}\right)}
\end{aligned}
$$


Hence, if we set

$$
\psi_{\ell}(\delta) \stackrel{\text { def }}{=} \max \left((1+\delta)^{r(\Lambda)}\left(1+\psi_{\ell-1}(\delta)\right)^{r\left(\Lambda^{\prime}\right)}\right)^{\frac{1}{r\left(\Lambda+\Lambda^{\prime}\right)}}-1,
$$

where the maximum is taken over all possible values of $r(\Lambda), r\left(\Lambda^{\prime}\right), r(\Lambda+$ $\left.\Lambda^{\prime}\right)$ then $\psi_{\ell}(\delta) \longrightarrow_{\delta \rightarrow 0} 0$ and (2.12) implies that $\Lambda+\Lambda^{\prime} \in \operatorname{Min}_{\psi_{\ell}(\delta)}(x)$ as desired. We take $\psi(\delta) \stackrel{\text { def }}{=} \max _{\ell=1}^{n} \psi_{\ell}(\delta)$. Now if $\ell>n$ one can find a subsequence $1 \leq i_{1}<i_{2} \cdots<i_{d} \leq n$ such that $r\left(\sum_{i=1}^{\ell} \Lambda_{i}\right)=r\left(\sum_{j=1}^{d} \Lambda_{i_{j}}\right)$ and in particular, $\sum_{j=1}^{d} \Lambda_{i_{j}}$ is of finite index in $\sum_{i=1}^{\ell} \Lambda_{i}$. From the first part of the argument we see that $\sum_{j=1}^{d} \Lambda_{i_{j}} \in \operatorname{Min}_{\psi(\delta)}(x)$ and as the covolume of $\sum_{i=1}^{\ell} \Lambda_{i}$ is not larger than that of $\sum_{j=1}^{d} \Lambda_{i_{j}}$ we deduce that $\sum_{i=1}^{\ell} \Lambda_{i} \in \operatorname{Min}_{\psi_{\ell}(\delta)}(x)$ as well.

To verify the last assertion, note that when $\operatorname{dim}_{\delta}(x)=n,(2.10)$ implies the existence of a finite index subgroup $x^{\prime}$ of $x$ belonging to $\operatorname{Min}_{\psi(\delta)}(x)$. In particular, $1 \leq\left|x^{\prime}\right|^{\frac{1}{n}} \leq(1+\psi(\delta)) \alpha(x)$ as desired.

Proof of Theorem 1.1 assuming Theorem 2.7. By Proposition 2.4 we may assume that $A x$ is bounded. Let $\varepsilon_{j} \in(0,1)$ so that $\varepsilon_{j} \rightarrow_{j} 0$. By Theorem 2.7 we know that $U_{n}^{x, \varepsilon_{j}} \neq \varnothing$. This means there is a sequence $a_{j} \in A$ such that $\operatorname{dim}_{\delta_{j}}\left(a_{j} x\right)=n$ where $\delta_{j}=n \varepsilon_{j} \rightarrow 0$. The sequence $\left\{a_{j} x\right\}$ is bounded, and hence has limit points, so passing to a subsequence we let $x^{\prime} \stackrel{\text { def }}{=} \lim a_{j} x$. By Lemma 2.8 we have

$$
1 \geq \limsup _{j} \alpha\left(a_{j} x\right) \geq \liminf _{j} \alpha\left(a_{j} x\right) \geq \lim _{j}\left(1+\psi\left(\delta_{j}\right)\right)^{-1}=1,
$$

which shows that $\lim _{j} \alpha\left(a_{j} x\right)=1$. The function $\alpha$ is continuous on $\mathcal{L}_{n}$ and therefore $\alpha\left(x^{\prime}\right)=1$, i.e. $x^{\prime} \in \overline{A x}$ is stable.

\section{Covers of Euclidean space}

In this section we will prove Theorem 2.7, thus completing the proof of Theorem 1.1. Our main tool will be McMullen's Theorem 3.3. Before stating it we introduce some terminology. We fix an invariant metric on $A$, and let $R>0$ and $k \in\{0, \ldots, n-1\}$.

Definition 3.1. We say that a subset $U \subset A$ is $(R, k)$-almost affine if it is contained in an $R$-neighborhood of a coset of a connected $k$ dimensional subgroup of $A$.

Definition 3.2. An open cover $\mathcal{U}$ of $A$ is said to have inradius $r>0$ if for any $a \in A$ there exists $U \in \mathcal{U}$ such that $B_{r}(a) \subset U$, where $B_{r}(a)$ denotes the ball in $A$ of radius $r$ around $a$. 
Theorem 3.3 (Theorem 5.1 of [McM05]). Let $\mathcal{U}$ be an open cover of $A$ with inradius $r>0$ and let $R>0$. Suppose that for any $1 \leq k \leq n-1$, every connected component $V$ of the intersection of $k$ distinct elements of $\mathcal{U}$ is $(R,(n-1-k))$-almost affine. Then there is a point in $A$ which belongs to at least $n$ distinct elements of $\mathcal{U}$. In particular, there are at least $n$ distinct non-empty sets in $\mathcal{U}$.

The hypotheses of McMullen's theorem were slightly weaker but the version above is sufficient for our purposes. We give a different proof of Theorem 3.3 in this paper; namely it follows from the more general Theorem 7.2, which is proved in Appendix A.

3.1. Verifying the hypotheses of Theorem 3.3. Below we fix a compact set $K \subset \mathcal{L}_{n}$ and a lattice $x$ for which $A x \subset K$. Furthermore, we fix $\varepsilon>0$ and denote the collection $\mathcal{U}^{x, \varepsilon}$ defined in (2.9) by $\mathcal{U}=$ $\left\{U_{i}\right\}_{i=1}^{n}$.

Lemma 3.4. The collection $\mathcal{U}$ forms an open cover of $A$ with positive inradius.

Proof. The fact that the sets $U_{i} \subset A$ are open follows readily from the requirement in (2.9) that $\operatorname{dim}_{\delta}$ is constant in a neighborhood of $\delta=k \varepsilon$. Given $a \in A$, let $1 \leq k_{0} \leq n$ be the minimal number $k$ for which $\operatorname{dim}_{\left(k+\frac{1}{2}\right) \varepsilon}(a x) \leq k$ (this inequality holds trivially for $k=n$ ). From the minimality of $k_{0}$ we conclude that $\operatorname{dim}_{\delta}(a x)=k_{0}$ for any $\delta \in\left[\left(k_{0}-\frac{1}{2}\right) \varepsilon,\left(k_{0}+\frac{1}{2}\right) \varepsilon\right]$. This shows that $a \in U_{k_{0}}$ so $\mathcal{U}$ is indeed a cover of $A$.

We now show that the cover has positive inradius. Let $W \subset G$ be the open neighborhood of the identity obtained from Lemma 2.6 for $\rho \stackrel{\text { def }}{=} \frac{\varepsilon}{2}$. Taking $\delta_{0} \stackrel{\text { def }}{=} k_{0} \varepsilon$ we find that for any $g \in W, \delta \in\left(\left(k_{0}-\frac{1}{4}\right) \varepsilon,\left(k_{0}+\frac{1}{4}\right) \varepsilon\right)$ we have that $\operatorname{dim}_{\delta}(\operatorname{gax})=k_{0}$. This shows that $(W \cap A) a \subset U_{k_{0}}$. Since $W \cap A$ is an open neighborhood of the identity in $A$ and the metric on $A$ is invariant under translation by elements of $A$, there exists $r>0$ (independent of $k_{0}$ and $a$ ) so that $B_{r}(a) \subset U_{k_{0}}$. In other words, the inradius of $\mathcal{U}$ is positive as desired.

The following will be used for verifying the second hypothesis of Theorem 3.3.

Lemma 3.5. There exists $R>0$ such that any connected component of $U_{k}$ is $(R, k-1)$-almost affine.

Definition 3.6. For a discrete subgroup $\Lambda \subset \mathbb{R}^{d}$ of rank $k$, let

$$
c(\Lambda) \stackrel{\text { def }}{=} \inf \left\{|a \Lambda|^{1 / k}: a \in A\right\},
$$


and say that $\Lambda$ is incompressible if $c(\Lambda)>0$.

Lemma 3.5 follows from:

Theorem 3.7 ([McM05, Theorem 6.1]). For any positive $c, C$ there exists $R>0$ such that if $\Lambda \subset \mathbb{R}^{n}$ is an incompressible discrete subgroup of rank $k$ with $c(\Lambda) \geq c$ then $\left\{a \in A:|a \Lambda|^{1 / k} \leq C\right\}$ is $(R, j)$-almost affine for some $j \leq \operatorname{gcd}(k, n)-1$.

Proof of Lemma 3.5. We first claim that there exists $c>0$ such that for any discrete subgroup $\Lambda \subset x$ we have that $c(\Lambda) \geq c$. To see this, recall that $A x$ is contained in a compact subset $K$, and hence by Mahler's compactness criterion, there is a positive lower bound on the length of any non-zero vector belonging to a lattice in $K$. On the other hand, Minkowski's convex body theorem shows that the shortest nonzero vector in a discrete subgroup $\Lambda \subset \mathbb{R}^{n}$ is bounded above by a constant multiple of $|\Lambda|^{1 / r(\Lambda)}$. This implies the claim.

In light of Theorem 3.7, it suffices to show that there is $C>0$ such that if $V \subset U_{k}$ is a connected component, then there exists $\Lambda \subset x$ such that $V \subset\left\{a \in A:|a \Lambda|^{1 / k} \leq C\right\}$. For any $1 \leq k \leq n$, write $\mathbf{g r}_{k}$ for the Grassmannian of $k$-dimensional subspaces of $\mathbb{R}^{n}$. Define

$$
\mathcal{M}: U_{k} \rightarrow \mathbf{g r}_{k}, \quad \mathcal{M}(a) \stackrel{\text { def }}{=} a^{-1} \mathbf{V}_{k \varepsilon}(a x)
$$

Observe that $\mathcal{M}$ is locally constant on $U_{k}$. Indeed, by definition of $U_{k}$, for $a_{0} \in U_{k}$ there exists $0<\rho<\frac{\varepsilon}{2}$ such that $\operatorname{dim}_{\delta}\left(a_{0} x\right)=k$ for any $\delta \in(k \varepsilon-\rho, k \varepsilon+\rho)$. Applying Lemma 2.6 for the lattice $a_{0} x$ with $\rho$ and $\delta_{0}=k \varepsilon$ we see that for any $a$ in a neighborhood of the identity in $A$,

$$
\mathcal{M}\left(a a_{0}\right)=a_{0}^{-1} a^{-1} \mathbf{V}_{k \varepsilon}\left(a a_{0} x\right)=a_{0}^{-1} \mathbf{V}_{k \varepsilon}\left(a_{0} x\right)=\mathcal{M}\left(a_{0}\right) .
$$

Now let $\Lambda \stackrel{\text { def }}{=} x \cap \mathcal{M}(a)$ where $a \in V ; \Lambda$ is well-defined since $\mathcal{M}$ is locally constant. Then for $a \in V$,

$$
a \Lambda=a(x \cap \mathcal{M}(a))=a\left(x \cap a^{-1} \mathbf{V}_{k \varepsilon}(a x)\right)=a x \cap \mathbf{V}_{k \varepsilon}(a x) .
$$

By Lemma 2.8 we have that

$$
|a \Lambda|^{1 / k}=\left|a x \cap \mathbf{V}_{k \varepsilon}(a x)\right|^{1 / k}<(1+\psi(k \varepsilon)) \alpha(a x) .
$$

Since $\alpha(a x) \leq 1$ we may take $C \stackrel{\text { def }}{=} 1+\psi(k \varepsilon)$ to complete the proof.

Proof of Theorem 2.7. Assume by contradiction that $A x$ is bounded but $U_{n}^{x, \varepsilon}=\varnothing$ for some $\varepsilon \in(0,1)$. Then by Lemma 3.4,

$$
\mathcal{U} \stackrel{\text { def }}{=}\left\{U_{1}, \ldots, U_{n-1}\right\}, \text { where } U_{j} \stackrel{\text { def }}{=} U_{j}^{x, \varepsilon},
$$


is a cover of $A$ of positive inradius. Moreover, if $V$ is a connected component of $U_{j_{1}} \cap \cdots \cap U_{j_{k}}$ with $j_{1}<\cdots<j_{k} \leq n-1$, then $V_{k} \subset U_{j_{1}}$ and $j_{1} \leq n-k$. So in light of Lemma 3.5, the hypotheses of Theorem 3.3 are satisfied. We deduce that $\mathcal{U}=\left\{U_{1}, \ldots, U_{n-1}\right\}$ contains at least $n$ elements, which is impossible.

\section{Bounds on Mordell's COnstant}

In analogy with (2.1) we define for any $x \in \mathcal{L}_{n}$ and $1 \leq k \leq n$,

$$
\begin{aligned}
& \mathcal{V}_{k}(x) \stackrel{\text { def }}{=}\left\{|\Lambda|^{1 / r(\Lambda)}: \Lambda \subset x, r(\Lambda)=k\right\}, \\
& \alpha_{k}(x) \stackrel{\text { def }}{=} \min \mathcal{V}_{k}(x) .
\end{aligned}
$$

The following is clearly a consequence of Theorem 1.1:

Corollary 4.1. For any $x \in \mathcal{L}_{n}$, any $\varepsilon>0$ and any $k \in\{1, \ldots, n\}$ there is $a \in A$ such that $\alpha_{k}(a x) \geq 1-\varepsilon$.

As the lattice $x=\mathbb{Z}^{n}$ shows, the constant 1 appearing in this corollary cannot be improved for any $k$. Note also that the case $k=1$ of Corollary 4.1, although not stated explicitly in [McM05], could be derived easily from McMullen's results in conjunction with [BSD56].

Proof of Corollary 1.2. Since the $A$-action maps a symmetric box $\mathcal{B}$ to a symmetric box of the same volume, the function $\kappa: \mathcal{L}_{n} \rightarrow \mathbb{R}$ in (1.1) is $A$-invariant. By the case $k=1$ of Corollary 4.1 , for any $\varepsilon>0$ and any $x \in \mathcal{L}_{n}$ there is $a \in A$ such that $a x$ does not contain nonzero vectors of Euclidean length at most $1-\varepsilon$, and hence does not contain nonzero vectors in the cube $\left[-\left(\frac{1}{\sqrt{n}}-\varepsilon\right),\left(\frac{1}{\sqrt{n}}-\varepsilon\right)\right]^{n}$. This implies that $\kappa(x) \geq\left(\frac{1}{\sqrt{n}}\right)^{n}$, as claimed.

We do not know whether the bound $\kappa_{n} \geq n^{-n / 2}$ is asymptotically optimal. However, it is not optimal for any fixed dimension $n$ :

Proposition 4.2. For any $n, \kappa_{n}>n^{-n / 2}$.

Proof. It is clear from the definition of the functions $\kappa$ and $\alpha_{k}$ that if $x_{j} \rightarrow x_{0}$ in $\mathcal{L}_{n}$, then

$$
\kappa\left(x_{0}\right) \leq \liminf _{j} \kappa\left(x_{j}\right) \text { and } \alpha_{k}\left(x_{0}\right) \geq \limsup _{j} \alpha_{k}\left(x_{j}\right) .
$$

A simple compactness argument implies that the infimum in (1.2) is attained, that is there is $x \in \mathcal{L}_{n}$ such that $\kappa_{n}=\kappa(x)$; moreover, for any $x_{0} \in \overline{A x}, \kappa\left(x_{0}\right)=\kappa(x)=\kappa_{n}$. Using the case $k=1$ of Corollary 4.1, we let $x_{0}$ be a stable lattice in $\overline{A x}$ such that $\alpha_{1}\left(x_{0}\right) \geq 1$. That is, $x_{0}$ 
contains no vectors in the open unit Euclidean ball, so the open cube $C \stackrel{\text { def }}{=}\left(-\frac{1}{\sqrt{n}}, \frac{1}{\sqrt{n}}\right)^{n}$ is admissible. Moreover, the only possible vectors in $x_{0}$ on $\partial C$ are on the corners of $C$, so there is $\varepsilon>0$ such that the box $C^{\prime} \stackrel{\text { def }}{=}\left(-\frac{1}{\sqrt{n}}, \frac{1}{\sqrt{n}}\right)^{n-1} \times\left(-\left(\frac{1}{\sqrt{n}}+\varepsilon\right), \frac{1}{\sqrt{n}}+\varepsilon\right)$ is also admissible. Taking closed boxes $\mathcal{B} \subset C^{\prime}$ with volume arbitrarily close to that of $C^{\prime}$, we see that

$$
\kappa_{n}=\kappa\left(x_{0}\right) \geq \frac{\operatorname{Vol}\left(C^{\prime}\right)}{2^{n}}>n^{-n / 2} .
$$

Our next goal is Corollary 4.6 which gives an explicit lower bound on $\kappa_{n}$, which improves (1.3) for $n$ congruent to $1 \bmod 4$. To obtain our bound we treat separately lattices with bounded or unbounded $A$-orbits. If $A x$ is unbounded we bound $\kappa(x)$ by using an inductive procedure and the work of Birch and Swinnerton-Dyer, as in §2.1. In the bounded case we use arguments of McMullen and known upper bounds for Hadamard's determinant problem. Our method applies with minor modifications whenever $n$ is not divisible by 4 . We begin with an analogue of Lemma 2.1.

Lemma 4.3. Suppose $x=[g] \in \mathcal{L}_{n}$ with $g$ in upper triangular block form as in (2.2). Then $\kappa(x) \geq \prod_{1}^{k} \kappa\left(\left[g_{i}\right]\right)$. In particular $\kappa(x) \geq$ $\prod_{1}^{k} n_{i}^{-n_{i} / 2}$.

Proof. By induction, it suffices to prove the Lemma in case $k=2$. In this case there is a direct sum decomposition $\mathbb{R}^{n}=V_{1} \oplus V_{2}$ where the $V_{i}$ are spanned by standard basis vectors, and if we write $\pi: \mathbb{R}^{n} \rightarrow V_{2}$ for the corresponding projection, then $\left[g_{1}\right]=x \cap V_{1},\left[g_{2}\right]=\pi(x)$. Write $\kappa^{(i)} \stackrel{\text { def }}{=} \kappa\left(\left[g_{i}\right]\right)$. Then for $\varepsilon>0$, there are symmetric boxes $\mathcal{B}_{i} \subset V_{i}$ such that $\mathcal{B}_{i}$ is admissible for $\left[g_{i}\right]$ and

$$
\operatorname{Vol}\left(\mathcal{B}_{i}\right) \geq \frac{\kappa^{(i)}-\varepsilon}{2^{n_{i}}}
$$

We claim that $\mathcal{B} \stackrel{\text { def }}{=} \mathcal{B}_{1} \times \mathcal{B}_{2}$ is admissible for $x$. To see this, suppose $u \in x \cap \mathcal{B}$. Since $\pi(u) \in \mathcal{B}_{2}$ and $\mathcal{B}_{2}$ is admissible for $\pi(x)=\left[g_{2}\right]$ we must have $\pi(u)=0$, i.e. $u \in x \cap V_{1}=\left[g_{1}\right]$; since $\mathcal{B}_{1}$ is admissible for $\left[g_{1}\right]$ we must have $u=0$.

This implies

$$
\kappa(x) \geq 2^{n} \operatorname{Vol}(\mathcal{B})=2^{n_{1}} \operatorname{Vol}\left(\mathcal{B}_{1}\right) \cdot 2^{n_{2}} \operatorname{Vol}\left(\mathcal{B}_{2}\right) \geq\left(\kappa^{(1)}-\varepsilon\right)\left(\kappa^{(2)}-\varepsilon\right),
$$

and the result follows taking $\varepsilon \rightarrow 0$. 
Corollary 4.4. If $x \in \mathcal{L}_{n}$ is such that $A x$ is unbounded then

$$
\kappa(x) \geq(n-1)^{-(n-1) / 2} .
$$

Proof. If $A x$ is unbounded then by [BSD56], up to a permutation of the axes, there is $x^{\prime} \in \overline{A x}$ so that $x^{\prime}=[g]$ is in upper triangular form, with $k \geq 2$ blocks. Let the corresponding parameters as in (2.2) be $n=n_{1}+\cdots+n_{k}$. Since $\kappa(x) \geq \kappa\left(x^{\prime}\right)$, by Lemma 4.3 it suffices to prove that

$$
\prod_{i=1}^{k} \frac{1}{n_{i}^{n_{i} / 2}} \geq \frac{1}{(n-1)^{\frac{n-1}{2}}} .
$$

It is easy to check that for $j=1, \ldots, n-1$,

$$
j^{\frac{j}{2}}(n-j)^{\frac{n-j}{2}} \leq(n-1)^{\frac{n-1}{2}},
$$

and the case $k=2$ of (4.4) follows. By induction on $k$ one then shows that $\prod_{i=1}^{k} n_{i}^{-n_{i} / 2} \geq(n-k+1)^{-\frac{n-k+1}{2}}$ and this implies (4.4) for all $k \geq 2$.

To treat the bounded orbits we will use known bounds on the Hadamard determinant problem, which we now recall. Let

$$
h_{n} \stackrel{\text { def }}{=} \sup \left\{\left|\operatorname{det}\left(a_{i j}\right)\right|: \forall i, j \in\{1, \ldots, n\},\left|a_{i j}\right| \leq 1\right\} .
$$

Hadamard showed that $h_{n} \leq n^{n / 2}$ and proved that this bound is not optimal unless $n$ is equal to 1,2 or is a multiple of 4 . Explicit upper bounds for such $n$ have been obtained by Barba, Ehlich and Wojtas (see $[\mathrm{BC} 72, \mathrm{Wik}])$.

Proposition 4.5. If $x \in \mathcal{L}_{n}$ has a bounded $A$-orbit then $\kappa(x) \geq \frac{1}{h_{n}}$.

Sketch of proof. Let $\varepsilon>0$. There is $p<\infty$ such that the $L^{p}$ norm and the $L^{\infty}$ norm on $\mathbb{R}^{n}$ are $1+\varepsilon$-biLipschitz; i.e. for any $v \in \mathbb{R}^{n}$,

$$
\frac{\|v\|_{p}}{1+\varepsilon} \leq\|v\|_{\infty} \leq(1+\varepsilon)\|v\|_{p}
$$

In [McM05], McMullen showed that the closure of any bounded $A$-orbit contains a well-rounded lattice, i.e. a lattice whose shortest nonzero vectors span $\mathbb{R}^{n}$. In McMullen's paper, the length of the shortest vectors was measured using the Euclidean norm, but McMullen's arguments apply equally well to the shortest vectors with respect to the $L^{p}$ norm. Thus there is $a \in A$ and vectors $v_{1}, \ldots, v_{n} \in a x$ spanning $\mathbb{R}^{n}$, such that for $i=1, \ldots, n$,

$$
\left\|v_{i}\right\| \in[r,(1+\varepsilon) r]
$$

Here $r$ is the length, with respect to the $L^{p}$-norm, of the shortest nonzero vector of $a x$. Using the two sides of (4.6) we find that $a x$ 
contains an admissible symmetric box of sidelength $r /(1+\varepsilon)$, and the $L^{\infty}$ norm of the $v_{i}$ is at most $(1+\varepsilon)^{2} r$. Let $A$ be the matrix whose columns are the $v_{i}$. Since the $v_{i}$ span $\mathbb{R}^{n}$, $\operatorname{det} A \neq 0$, and since $x$ is unimodular, $|\operatorname{det} A| \geq 1$. Recalling (4.5) we find that

$$
\left.1 \leq|\operatorname{det} A| \leq\left((1+\varepsilon)^{2}\right) r\right)^{n} h_{n},
$$

and by definition of $\kappa$ we find

$$
\kappa(x)=\kappa(a x) \geq\left(\frac{r}{1+\varepsilon}\right)^{n} .
$$

Putting these together and letting $\varepsilon \rightarrow 0$ we see that $\kappa(x) \geq \frac{1}{h_{n}}$, as claimed.

Corollary 4.6. If $n \geq 5$ is congruent to $1 \bmod 4$, then

$$
\kappa_{n} \geq \frac{1}{\sqrt{2 n-1}(n-1)^{(n-1) / 2}} .
$$

Proof. The right hand side of (4.7) is clearly smaller than the right hand side of (4.3). Now the claim follows from Corollary 4.4 and Proposition 4.5, using Barba's bound

$$
h_{n} \leq \sqrt{2 n-1}(n-1)^{(n-1) / 2} .
$$

The same argument applies in the other cases in which $n$ is sufficiently large and is not divisible by 4 , since in these cases there are explicit upper bounds for the numbers $h_{n}$ which could be used in place (4.8).

\section{Two Strategies For Minkowski's CONJECTURE}

We begin by recalling the well-known Davenport-Remak strategy for proving Minkowski's conjecture. The function $N(u)=\prod_{1}^{n} u_{i}$ is clearly $A$-invariant, and it follows that the quantity

$$
\widetilde{N}(x) \stackrel{\text { def }}{=} \sup _{u \in \mathbb{R}^{n}} \inf _{v \in x}|N(u-v)|
$$

appearing in (1.4) is $A$-invariant. Moreover, it is easy to show that if $x_{n} \rightarrow x$ in $\mathcal{L}_{n}$ then $\widetilde{N}(x) \geq \lim \sup _{n} \tilde{N}\left(x_{n}\right)$. Therefore, in order to show the estimate (1.4) for $x^{\prime} \in \mathcal{L}_{n}$, it is enough to show it for some $x \in \overline{A x^{\prime}}$. Suppose that $x$ satisfies (1.5) with $d=n$; that is for every 
$u \in \mathbb{R}^{n}$ there is $v \in x$ such that $\|u-v\| \leq \frac{\sqrt{n}}{2}$. Then applying the inequality of arithmetic and geometric means one finds

$$
\prod_{1}^{n}\left(\left|u_{i}-v_{i}\right|^{2}\right)^{\frac{1}{n}} \leq \frac{1}{n} \sum_{1}^{n}\left|u_{i}-v_{i}\right|^{2} \leq \frac{1}{4}
$$

which implies $|N(u-v)| \leq \frac{1}{2^{n}}$. The upshot is that in order to prove Minkowski's conjecture, it is enough to prove that for every $x^{\prime} \in \mathcal{L}_{n}$ there is $x \in \overline{A x}$ satisfying (1.5). So in light of Theorem 1.1 we obtain:

Corollary 5.1. If all stable lattices in $\mathcal{L}_{n}$ satisfy (1.5), then Minkowski's conjecture is true in dimension $n$.

In the next two subsections, we outline two strategies for establishing that all stable lattices satisfy (1.5). Both strategies yield affirmative answers in dimensions $n \leq 7$, thus providing new proofs of Minkowski's conjecture in these dimensions.

5.1. Using Korkine-Zolotarev reduction. Korkine-Zolotarev reduction is a classical method for choosing a basis $v_{1}, \ldots, v_{n}$ of a lattice $x \in \mathcal{L}_{n}$. Namely one takes for $v_{1}$ a shortest nonzero vector of $x$ and denotes its length by $A_{1}$. Then, proceeding inductively, for $v_{i}$ one takes a vector whose projection onto $\left(\operatorname{span}\left(v_{1}, \ldots, v_{i-1}\right)\right)^{\perp}$ is shortest (among those with nonzero projection), and denotes the length of this projection by $A_{i}$. In case there is more than one shortest vector the process is not uniquely defined. Nevertheless we call $A_{1}, \ldots, A_{n}$ the diagonal $K Z$ coefficients of $x$ (with the understanding that these may be multiply defined for some measure zero subset of $\mathcal{L}_{n}$ ). Since $x$ is unimodular we always have

$$
\prod A_{i}=1 \text {. }
$$

Korkine and Zolotarev proved the bounds

$$
A_{i+1}^{2} \geq \frac{3}{4} A_{i}^{2}, \quad A_{i+2}^{2} \geq \frac{2}{3} A_{i}^{2} .
$$

A method introduced by Woods [Woo65] and developed further in [HGRS09] leads to an upper bound on $\operatorname{covrad}(x)$ in terms of the diagonal KZ coefficients. The method relies on the following estimate. Below $\gamma_{n} \stackrel{\text { def }}{=} \sup _{x \in \mathcal{L}_{n}} \alpha_{1}(x)$, where $\alpha_{1}$ is defined via $(4.1)$, that is, $\gamma_{n}^{2}$ is the so-called Hermite constant.

Lemma 5.2 (Woods). Suppose that $x$ is a lattice in $\mathbb{R}^{n}$ of covolume $d$, and suppose that $2 A_{1}^{n} \geq d \gamma_{n+1}^{n+1}$. Then

$$
\operatorname{covrad}^{2}(x) \leq A_{1}^{2}-\frac{A_{1}^{2 n+2}}{d^{2} \gamma_{n+1}^{2 n+2}} .
$$


Woods also used the following observation:

Lemma 5.3. Let $x$ be a lattice in $\mathbb{R}^{n}$, let $\Lambda$ be a subgroup, and let $\Lambda^{\prime}$ denote the projection of $x$ onto $(\operatorname{span} \Lambda)^{\perp}$. Then

$$
\operatorname{covrad}^{2}(x) \leq \operatorname{covrad}^{2}(\Lambda)+\operatorname{covrad}^{2}\left(\Lambda^{\prime}\right)
$$

As a consequence of Lemmas 5.2 and 5.3, we obtain:

Proposition 5.4. Suppose $A_{1}, \ldots, A_{n}$ are diagonal KZ coefficients of $x \in \mathcal{L}_{n}$ and suppose $n_{1}, \ldots, n_{k}$ are positive integers with $n=n_{1}+\cdots+$ $n_{k}$. Set

$$
m_{i} \stackrel{\text { def }}{=} n_{1}+\cdots+n_{i} \text { and } d_{i} \stackrel{\text { def }}{=} \prod_{j=m_{i-1}+1}^{m_{i}} A_{j}
$$

If

$$
2 A_{m_{i-1}+1} \geq d_{i} \gamma_{n_{i}+1}^{n_{i}+1}
$$

for each $i$, then

$$
\operatorname{covrad}^{2}(x) \leq \sum_{i=1}^{k}\left(A_{m_{i-1}+1}^{2}-\frac{A_{m_{i-1}+1}^{2 n_{i}+2}}{d_{i}^{2} \gamma_{n_{i}+1}^{n_{i}+1}}\right)
$$

Proof. Let $v_{1}, \ldots, v_{n}$ be the basis of $x$ obtained by the Korkine Zolotarev reduction process. Let $\Lambda_{1}$ be the subgroup of $x$ generated by $v_{1}, \ldots, v_{n_{1}}$, and for $i=2, \ldots, k$ let $\Lambda_{i}$ be the projection onto $\left(\bigoplus_{1}^{i-1} \Lambda_{j}\right)^{\perp}$ of the subgroup of $x$ generated by $v_{m_{i-1}+1}, \ldots, v_{m_{i}}$. This is a lattice of dimension $m_{i}$, and arguing as in the proof of (2.3) we see that it has covolume $d_{i}$. The assumption (5.4) says that we may apply Lemma 5.2 to each $\Lambda_{i}$. We obtain

$$
\operatorname{covrad}^{2}\left(\Lambda_{i}\right) \leq A_{m_{i-1}+1}^{2}-\frac{A_{m_{i-1}+1}^{2 n_{i}+2}}{d_{i}^{2} \gamma_{n_{i}+1}^{n_{i}+1}}
$$

for each $i$, and we combine these estimates using Lemma 5.3 and an obvious induction.

Remark 5.5. Note that it is an open question to determine the numbers $\gamma_{n}$; however, if we have a bound $\tilde{\gamma}_{n} \geq \gamma_{n}$ we may substitute it into Proposition 5.4 in place of $\gamma_{n}$, as this only makes the requirement (5.4) stricter and the conclusion (5.5) weaker.

Our goal is to apply this method to the problem of bounding the covering radius of stable lattices. We note:

Proposition 5.6. If $x$ is stable then we have the inequalities

$$
A_{1} \geq 1, \quad A_{1} A_{2} \geq 1, \quad \ldots \quad A_{1} \cdots A_{n-1} \geq 1 .
$$


Proof. In the above terms, the number $A_{1} \cdots A_{i}$ is equal to $|\Lambda|$ where $\Lambda$ is the subgroup of $x$ generated by $v_{1}, \ldots, v_{i}$.

This motivates the following:

Definition 5.7. We say that an $n$-tuple of positive real numbers $A_{1}, \ldots, A_{n}$ is $K Z$ stable if the inequalities (5.1), (5.2), (5.6) are satisfied. We denote the set of KZ stable $n$-tuples by KZS.

Note that KZS is a compact subset of $\mathbb{R}^{n}$. Recall that a composition of $n$ is an ordered $k$-tuple $\left(n_{1}, \ldots, n_{k}\right)$ of positive integers, such that $n=n_{1}+\ldots+n_{k}$. As an immediate application of Corollary 5.1 and Propositions 5.4 and 5.6 we obtain:

Theorem 5.8. For each composition $\mathcal{I} \stackrel{\text { def }}{=}\left(n_{1}, \ldots, n_{k}\right)$ of $n$, define $m_{i}, d_{i}$ by (5.3) and let $\mathcal{W}(\mathcal{I})$ denote the set

$$
\begin{aligned}
& \left\{\left(A_{1}, \ldots, A_{n}\right): \forall i,(5.4) \text { holds, and } \sum_{i=1}^{k}\left(A_{m_{i-1}+1}^{2}-\frac{A_{m_{i-1}+1}^{2 n_{i}+2}}{d_{i}^{2} \gamma_{n_{i}+1}^{n_{i}+1}}\right) \leq \frac{n}{4}\right\} . \\
& \text { If } \\
& \qquad \mathrm{KZS} \subset \bigcup_{\mathcal{I}} \mathcal{W}(\mathcal{I})
\end{aligned}
$$

then Minkowski's conjecture holds in dimension $n$.

Rajinder Hans-Gill has informed the authors that using arguments as in [HGRS09, HGRS11], it is possible to verify (5.7) in dimensions up to 7 , thus reproving Minkowski's conjecture in these dimensions.

5.2. Local maxima of covrad. The aim of this subsection is to prove Corollary 1.3, which shows that in order to establish that all stable lattices in $\mathbb{R}^{n}$ satisfy the covering radius bound (1.5), it suffices to check this on a finite list of lattices in each dimension $d \leq n$.

The function covrad : $\mathcal{L}_{n} \rightarrow \mathbb{R}$ may have local maxima, in the usual sense; that is, lattices $x \in \mathcal{L}_{n}$ for which there is a neighborhood $\mathcal{U}$ of $x$ in $\mathcal{L}_{n}$ such that for all $x^{\prime} \in \mathcal{U}$ we have $\operatorname{covrad}\left(x^{\prime}\right) \leq \operatorname{covrad}(x)$. DutourSikirić, Schürmann and Vallentin [DSSV12] gave a geometric characterization of lattices which are local maxima of the function covrad, and showed that there are finitely many in each dimension. Corollary 1.3 asserts that Minkowski's conjecture would follow if all local maxima of covrad satisfy the bound (1.5).

Proof of Corollary 1.3. We prove by induction on $n$ that any stable lattice satisfies the bound (1.5) and apply Corollary 5.1. Let $\mathcal{S}$ denote the set of stable lattices in $\mathcal{L}_{n}$. It is compact so the function covrad attains a maximum on $\mathcal{S}$, and it suffices to show that this maximum is 
at most $\frac{\sqrt{n}}{2}$. Let $x \in \mathcal{S}$ be a point at which the maximum is attained. If $x$ is an interior point of $\mathcal{S}$ then necessarily $x$ is a local maximum for covrad and the required bound holds by hypothesis. Otherwise, there is a sequence $x_{j} \rightarrow x$ such that $x_{j} \in \mathcal{L}_{n} \backslash \mathcal{S}$; thus each $x_{j}$ contains a discrete subgroup $\Lambda_{j}$ with $\left|\Lambda_{j}\right|<1$ and $r\left(\Lambda_{j}\right)<n$. Passing to a subsequence we may assume that that $r\left(\Lambda_{j}\right)=k<n$ is the same for all $j$, and $\Lambda_{j}$ converges to a discrete subgroup $\Lambda$ of $x$. Since $x$ is stable we must have $|\Lambda|=1$. Let $\pi: \mathbb{R}^{n} \rightarrow(\operatorname{span} \Lambda)^{\perp}$ by the orthogonal projection and let $\Lambda^{\prime} \stackrel{\text { def }}{=} \pi(x)$.

It suffices to show that both $\Lambda$ and $\Lambda^{\prime}$ are stable. Indeed, if this holds then by the induction hypothesis, both $\Lambda$ and $\Lambda^{\prime}$ satisfy (1.5) in their respective dimensions $k, n-k$, and by Lemma 5.3 , so does $x$. To see that $\Lambda$ is stable, note that any subgroup $\Lambda_{0} \subset \Lambda$ is also a subgroup of $x$, and since $x$ is stable, it satisfies $\left|\Lambda_{0}\right| \geq 1$. To see that $\Lambda^{\prime}$ is stable, note that if $\Lambda_{0} \subset \Lambda^{\prime}$ then $\widetilde{\Lambda_{0}} \stackrel{\text { def }}{=} x \cap \pi^{-1}\left(\Lambda_{0}\right)$ is a discrete subgroup of $x$ so satisfies $\left|\widetilde{\Lambda_{0}}\right| \geq 1$. Since $|\Lambda|=1$ and $\pi$ is orthogonal, we argue as in the proof of (2.3) to obtain

$$
1 \leq\left|\widetilde{\Lambda_{0}}\right|=|\Lambda| \cdot\left|\Lambda_{0}\right|=\left|\Lambda_{0}\right|
$$

so $\Lambda^{\prime}$ is also stable, as required.

In [DSSV12], it was shown that there is a unique local maximum for covrad in dimension 1 , none in dimensions 2-5, and a unique one in dimension 6. Local maxima of covrad in dimension 7 are classified in the manuscript [DS13]; there are 2 such lattices. Thus in total, in dimensions $n \leq 7$ there are 4 local maxima of the function covrad. We were informed by Mathieu Dutour-Sikiric that these lattices all satisfy the covering radius bound (1.5). Thus Corollary 1.3 yields another proof of Minkowski's conjecture, in dimensions $n \leq 7$. In [DSSV12, §7], an infinite list of lattices (denote there by $\left[L_{n}, Q_{n}\right]$ ) is defined. The list consists of one lattice in each dimension $n \geq 6$, each of which is a local maximum for the function covrad, and satisfies the bound (1.5). It is expected that for each $n$, this lattice has the largest covering radius among all local maxima in dimension $n$. In light of Corollary 1.3, the validity of the latter assertion would imply Minkowski's conjecture in all dimensions.

\section{A volume COMPUTATion}

The goal of this section is the following. 
Theorem 6.1. Let $m$ denote the $G$-invariant probability measure on $\mathcal{L}_{n}$ derived from Haar measure on $G$, and let $\mathcal{S}^{(n)}$ denote the subset of stable lattices in $\mathcal{L}_{n}$. Then $m\left(\mathcal{S}^{(n)}\right) \longrightarrow 1$ as $n \rightarrow \infty$.

Recalling the notation (4.1), for $k=1, \ldots, n-1$, let

$$
\mathcal{S}_{k}^{(n)}(t) \stackrel{\text { def }}{=}\left\{x \in \mathcal{L}_{n}: \alpha_{k}(x) \geq t\right\}, \quad \mathcal{S}_{k}^{(n)} \stackrel{\text { def }}{=} \mathcal{S}_{k}^{(n)}(1) .
$$

It is clear that $\mathcal{S}^{(n)}=\bigcap_{k=1}^{n-1} \mathcal{S}_{k}^{(n)}$. In order to prove Theorem 6.1 it is enough to prove that

$$
\max _{k=1, \ldots, n-1} m\left(\mathcal{L}_{n} \backslash \mathcal{S}_{k}^{(n)}\right)=o\left(\frac{1}{n}\right)
$$

as this implies

$$
\begin{aligned}
m\left(\mathcal{S}^{(n)}\right) & =1-m\left(\mathcal{L}_{n} \backslash \cap_{k=1}^{n-1} \mathcal{S}_{k}^{(n)}\right)=1-m\left(\cup_{k=1}^{n-1}\left(\mathcal{L}_{n} \backslash \mathcal{S}_{k}^{(n)}\right)\right) \\
& \geq 1-\sum_{k=1}^{n-1} m\left(\mathcal{L}_{n} \backslash \mathcal{S}_{k}^{(n)}\right)=1-(n-1) o\left(\frac{1}{n}\right) \stackrel{n \rightarrow \infty}{\longrightarrow} 1 .
\end{aligned}
$$

We will actually prove a bound which is stronger than (6.1), namely:

Proposition 6.2. There is $C_{1}>0$ such that if we set

$$
t_{k}=t(n, k) \stackrel{\text { def }}{=}\left(\frac{n}{C_{1}}\right)^{\frac{k(n-k)}{2 n}}
$$

then

$$
\max _{k=1, \ldots, n-1} m\left(\mathcal{L}_{n} \backslash \mathcal{S}_{k}^{(n)}\left(t_{k}\right)\right)=o\left(\frac{1}{n}\right) .
$$

In particular, $m\left(\bigcap_{k=1}^{n-1} \mathcal{S}_{k}^{(n)}\left(t_{k}\right)\right) \rightarrow_{n \rightarrow \infty} 1$.

Let

$$
\gamma_{n, k} \stackrel{\text { def }}{=} \sup _{x \in \mathcal{L}_{n}} \alpha_{k}(x)
$$

Recall that Rankin's constants or the generalized Hermite's constants, are defined as $\gamma_{n, k}^{2}$ (note that our notations differ from traditional notations by a square root). Thunder [Thu98] computed upper and lower bounds on $\gamma_{n, k}$ and in particular established the growth rate of $\gamma_{n, k}$. The numbers $t(n, k)$ have the same growth rate. Thus Proposition 6.2 should be interpreted as saying that the lattices in $\mathcal{L}_{n}$ for which the value of each $\alpha_{k}$ is close to the maximum possible value, occupy almost all of the measure of $\mathcal{L}_{n}$.

The proof of Proposition 6.2 relies on Thunder's work, which in turn was based on a variant of Siegel's formula [Sie45] which relates the 
Lebesgue measure on $\mathbb{R}^{n}$ and the measure $m$ on $\mathcal{L}_{n}$. We now review Siegel's method and Thunder's results.

In the sequel we consider $n \geq 2$ and $k \in\{1, \ldots, n-1\}$ as fixed and omit, unless there is risk of confusion, the symbols $n$ and $k$ from the notation. Consider the (set valued) map $\Phi=\Phi_{k}^{(n)}$ that assigns to each lattice $x \in \mathcal{L}_{n}$ the following subset of $\wedge^{k} \mathbb{R}^{n}$ :

$$
\Phi(x) \stackrel{\text { def }}{=}\left\{ \pm w_{\Lambda}: \Lambda \subset x \text { a primitive subgroup with } r(\Lambda)=k\right\},
$$

where $w_{\Lambda} \stackrel{\text { def }}{=} v_{1} \wedge \cdots \wedge v_{k}$ and $\left\{v_{i}\right\}_{1}^{k}$ forms a basis for $\Lambda$ (note that $w_{\Lambda}$ is well defined up to sign, and $\Phi(x)$ contains both possible choices). Let

$$
\mathscr{V}=\mathscr{V}_{k}^{(n)} \stackrel{\text { def }}{=}\left\{v_{1} \wedge \cdots \wedge v_{k}: v_{i} \in \mathbb{R}^{n}\right\} \backslash\{0\}
$$

be the variety of pure tensors in $\wedge^{k} \mathbb{R}^{n}$. For any a compactly supported bounded Riemann integrable function $f$ on $\mathscr{V}$ set

$$
\hat{f}: \mathcal{L}_{n} \rightarrow \mathbb{R}, \quad \hat{f}(x) \stackrel{\text { def }}{=} \sum_{w \in \Phi(x)} f(w) .
$$

Then it is known (see [Wei82]) that the (finite) sum (6.3) defines a function in $L^{1}\left(\mathcal{L}_{n}, m\right)$. Let $\theta=\theta_{k}^{(n)}$ denote the Radon measure on $\mathscr{V}$ defined by

$$
\int_{\mathscr{V}} f d \theta \stackrel{\text { def }}{=} \int_{\mathcal{L}_{n}} \hat{f} d m, \text { for } f \in C_{c}(\mathscr{V}) .
$$

In this section we write $G=G_{n} \stackrel{\text { def }}{=} \mathrm{SL}_{n}(\mathbb{R})$. There is a natural transitive action of $G_{n}$ on $\mathscr{V}$ and the stabilizer of $e_{1} \wedge \cdots \wedge e_{k}$ is the subgroup

$$
H=H_{k}^{(n)} \stackrel{\text { def }}{=}\left\{\left(\begin{array}{cc}
A & B \\
0 & D
\end{array}\right) \in G: A \in G_{k}, D \in G_{n-k}\right\} .
$$

We therefore obtain an identification $\mathscr{V} \simeq G / H$ and view $\theta$ as a measure on $G / H$.

It is well-known (see e.g. [Rag72]) that up to a proportionality constant there exists a unique $G$-invariant measure $m_{G / H}$ on $G / H$; moreover, given Haar measures $m_{G}, m_{H}$ on $G$ and $H$ respectively, there is a unique normalization of $m_{G / H}$ such that for any $f \in L^{1}\left(G, m_{G}\right)$

$$
\int_{G} f d m_{G}=\int_{G / H} \int_{H} f(g h) d m_{H}(h) d m_{G / H}(g H) .
$$

We choose the Haar measure $m_{G}$ so that it descends to our probability measure $m$ on $\mathcal{L}_{n}$; similarly, we choose the Haar measure $m_{H}$ so that the periodic orbit $H \mathbb{Z}^{n} \subset \mathcal{L}_{n}$ has volume 1 . These choices of Haar measures determine our measure $m_{G / H}$ unequivocally. It is clear from the defining formula (6.4) that $\theta$ is $G$-invariant and therefore the two 
measures $m_{G / H}, \theta$ are proportional. In fact (see [Sie45] for the case $k=1$ and [Wei82] for the general case),

$$
m_{G / H}=\theta \text {. }
$$

For $t>0$, let $\chi=\chi_{t}: \mathscr{V} \rightarrow \mathbb{R}$ be the restriction to $\mathscr{V}$ of the characteristic function of the ball of radius $t$ around the origin, in $\wedge^{k} \mathbb{R}^{n}$. Note that $\hat{\chi}(x)=0$ if and only if $x \in \mathcal{S}_{k}^{(n)}(t)$ and furthermore, $\hat{\chi}(x) \geq 1$ if $x \in \mathcal{L}_{n} \backslash \mathcal{S}_{k}^{(n)}(t)$. It follows that

$$
m\left(\mathcal{L}_{n} \backslash \mathcal{S}_{k}^{(n)}(t)\right) \leq \int_{\mathcal{L}_{n}} \widehat{\left(\chi_{t}\right)} d m=\int_{\mathscr{V}} \chi_{t} d \theta
$$

Let $V_{j}$ denote the volume of the Euclidean unit ball in $\mathbb{R}^{j}$ and let $\zeta$ denote the Riemann zeta function. We will use an unconventional convention $\zeta(1)=1$, which will make our formulae simpler. For $j \geq 1$, define

$$
R(j) \stackrel{\text { def }}{=} \frac{j^{2} V_{j}}{\zeta(j)}
$$

and

$$
B(n, k) \stackrel{\text { def }}{=} \frac{\prod_{j=1}^{n} R(j)}{\prod_{j=1}^{k} R(j) \prod_{j=1}^{n-k} R(j)} .
$$

The following calculation was carried out in [Thu98].

Theorem 6.3 (Thunder). For $t>0$, we have

$$
\int_{\mathscr{V}} \chi_{t} d m_{G / H}=B(n, k) \frac{t^{n}}{n} \text {. }
$$

We will need to bound $B(n, k)$.

Lemma 6.4. There is $C>0$ so that for all large enough $n$ and all $k=1, \ldots, n-1$,

$$
B(n, k) \leq\left(\frac{C}{n}\right)^{\frac{k(n-k)}{2}} .
$$

Proof. In this proof $c_{0}, c_{1}, \ldots$ are constants independent of $n, k, j$. Because of the symmetry $B(n, k)=B(n, n-k)$ it is enough to prove (6.8) with $k \leq \frac{n}{2}$. Using the formula $V_{j}=\frac{\pi^{j / 2}}{\Gamma\left(\frac{j}{2}+1\right)}$ we obtain

$$
\begin{aligned}
B(n, k) & =\prod_{j=1}^{k} \frac{R(n-k+j)}{R(j)}=\prod_{j=1}^{k} \frac{\zeta(j)(n-k+j)^{2} \frac{\pi^{(n-k+j) / 2}}{\Gamma\left(\frac{n-k+j}{2}+1\right)}}{\zeta(n-k+j) j^{2} \frac{\pi^{j / 2}}{\Gamma\left(\frac{j}{2}+1\right)}} \\
& =\prod_{j=1}^{k} \frac{\zeta(j)}{\zeta(n-k+j)} \cdot\left(\frac{n-k+j}{j}\right)^{2} \cdot \pi^{\frac{n-k}{2}} \cdot \frac{\Gamma\left(\frac{j}{2}+1\right)}{\Gamma\left(\frac{n-k+j}{2}+1\right)}
\end{aligned}
$$


Note that $\zeta(s) \geq 1$ is a decreasing function of $s>1$, so (recalling our convention $\zeta(1)=1) \frac{\zeta(j)}{\zeta(n-k+j)} \leq c_{0} \stackrel{\text { def }}{=} \zeta(2)$. It follows that for all large enough $n$ and for any $1 \leq j \leq k$,

$$
\frac{\zeta(j)}{\zeta(n-k+j)} \cdot\left(\frac{n-k+j}{j}\right)^{2} \cdot \pi^{\frac{n-k}{2}} \leq c_{0} n^{2} \pi^{\frac{n-k}{2}} \leq 4^{\frac{n-k}{2}} .
$$

According to Stirling's formula, there are positive constants $c_{1}, c_{2}$ such that for all $x \geq 2$,

$$
c_{1} \sqrt{\frac{2 \pi}{x}}\left(\frac{x}{e}\right)^{x} \leq \Gamma(x) \leq c_{2} \sqrt{\frac{2 \pi}{x}}\left(\frac{x}{e}\right)^{x} .
$$

We set $u \stackrel{\text { def }}{=} \frac{j}{2}+1$ and $v \stackrel{\text { def }}{=} \frac{n-k}{2}$, so that $u+v \geq \frac{n-1}{4}$, and obtain

$$
\begin{aligned}
\frac{\Gamma\left(\frac{j}{2}+1\right)}{\Gamma\left(\frac{n-k+j}{2}+1\right)} & =\frac{\Gamma(u)}{\Gamma(u+v)} \leq \frac{c_{2}}{c_{1}} \sqrt{\frac{u+v}{u}} \frac{u^{u}}{(u+v)^{u+v}} \frac{e^{u+v}}{e^{u}} \\
& \leq c_{3} e^{v} \frac{u^{u-1 / 2}}{(u+v)^{u+v-1 / 2}}=c_{3}\left(\frac{e}{u+v}\right)^{v} \frac{1}{\left(1+\frac{v}{u}\right)^{u-1 / 2}} \\
& \leq c_{3}\left(\frac{4 e}{n-1}\right)^{\frac{n-k}{2}} .
\end{aligned}
$$

Using (6.9) and (6.10) we obtain

$$
B(n, k) \leq\left[c_{3} 4^{\frac{n-k}{2}}\left(\frac{4 e}{n-1}\right)^{\frac{n-k}{2}}\right]^{k}=\left[c_{3}\left(\frac{16 e}{n-1}\right)^{\frac{n-k}{2}}\right]^{k} .
$$

So taking $C>16 c_{3} e$ we obtain (6.8) for all large enough $n$.

Proof of Proposition 6.2. Let $C$ be as in Lemma 6.4 and let $C_{1}>C$. Then by (6.7), (6.6) and Theorem 6.3, for all sufficiently large $n$ we have

$$
\begin{aligned}
m\left(\mathcal{L}_{n} \backslash \mathcal{S}_{k}^{(n)}\left(t_{k}\right)\right) & \leq B(n, k) \frac{t_{k}^{n}}{n} \\
& \leq \frac{1}{n}\left(\frac{C}{n}\right)^{\frac{k(n-k)}{2}}\left(\frac{n}{C_{1}}\right)^{\frac{k(n-k)}{2}}=\frac{1}{n}\left(\frac{C}{C_{1}}\right)^{\frac{k(n-k)}{2}} .
\end{aligned}
$$

Multiplying by $n$ and taking the maximum over $k$ we obtain

$$
n \max _{k=1, \ldots, n} m\left(\mathcal{L}_{n} \backslash \mathcal{S}_{k}^{(n)}\left(t_{k}\right)\right) \leq\left(\frac{C}{C_{1}}\right)^{\frac{n-1}{2}} \rightarrow_{n \rightarrow \infty} 0 .
$$




\section{Closed $A$-orbits and Well-rounded lattices}

It is an immediate consequence of Theorem 1.1 that any closed $A$ orbit contains a stable lattice. The purpose of this section is to show that the same is true for the set of well-rounded lattices. Note that this was proved by McMullen for compact orbits but for general closed orbits, does not follow from his results. Our proof relies on previous work of Tomanov and the second-named author [TW03], on [SW], and on a covering result (communicated to the authors by Michael Levin), whose proof is given in the appendix to this paper.

Theorem 7.1. For any $n$, any closed orbit $A x \subset \mathcal{L}_{n}$ contains a wellrounded lattice.

We will require the following topological result which generalizes Theorem 3.3. Let $s, t$ be natural numbers, and let $\Delta$ denote the $s$ dimensional simplex, which we think of concretely as $\operatorname{conv}\left(\mathbf{e}_{1}, \ldots, \mathbf{e}_{s+1}\right)$. We will discuss covers of $M \stackrel{\text { def }}{=} \Delta \times \mathbb{R}^{t}$, and give conditions guaranteeing that such a cover must cover a point at least $s+t+1$ times. For $j=1, \ldots, s+1$ let $F_{j}$ be the face of $\Delta$ opposite to $\mathbf{e}_{j}$, that is $F_{j}=\operatorname{conv}\left(\mathbf{e}_{i}: i \neq j\right)$. Also let $M_{j} \stackrel{\text { def }}{=} F_{j} \times \mathbb{R}^{t}$ be the corresponding subset of $M$.

Theorem 7.2. Suppose that $\mathcal{U}$ is a cover of $M$ by open sets satisfying the following conditions:

(i) For any connected component $U$ of any element of $\mathcal{U}$ there exists $j$ such that $U \cap M_{j}=\varnothing$.

(ii) There is $R$ so that for any connected component $U$ of the intersection of $k \leq s+t$ distinct elements of $\mathcal{U}$, the projection of $U$ to $\mathbb{R}^{t}$ is $(R, s+t-k)$-almost affine.

Then there is a point of $M$ which is covered at least $s+t+1$ times.

Note that hypothesis (ii) is trivially satisfied when $k \leq s$, since any subset of $\mathbb{R}^{t}$ is $(1, t)$-almost affine. Note also that Theorem 3.3 is the case $s=0$ of this statement. We give the proof of Theorem 7.2 in the appendix.

We will need some preparations in order to deduce Theorem 7.1 from Theorem 7.2. For $1 \leq d \leq n$, let

$$
\mathbf{I}_{d}^{n} \stackrel{\text { def }}{=}\left\{1 \leq i_{1}<\cdots<i_{d} \leq n\right\}
$$

denote the collection of multi-indices of length $d$ and for $J=\left(i_{1}, \ldots, i_{d}\right) \in$ $\mathbf{I}_{d}^{n}$ let $e_{J} \stackrel{\text { def }}{=} e_{i_{1}} \wedge \cdots \wedge e_{i_{d}}$. We equip $\bigwedge_{1}^{d} \mathbb{R}^{n}$ with the inner product with respect to which $\left\{e_{J}\right\}$ is an orthonormal basis, and denote by $\mathcal{E}_{d, n}$ the 
quotient of $\bigwedge_{1}^{d} \mathbb{R}^{n}$ by the equivalence relation $w \sim-w$. Note that the product of an element of $\mathcal{E}_{d, n}$ with a positive scalar is well-defined. We will (somewhat imprecisely) refer to elements of $\mathcal{E}_{d, n}$ as vectors. Given a subspace $L \subset \mathbb{R}^{n}$ with $\operatorname{dim} L=d$, we denote by $w_{L} \in \mathcal{E}_{d, n}$ the image of a vector of norm one in $\bigwedge_{1}^{d} L$. If $\Lambda \subset \mathbb{R}^{n}$ is a discrete subgroup of rank $d$, we denote by $w_{\Lambda} \in \mathcal{E}_{d, n}$ the image of the vector $v_{1} \wedge \cdots \wedge v_{d}$, where $\left\{v_{i}\right\}_{1}^{d}$ forms a basis for $\Lambda$. The reader may verify that these vectors are well-defined and satisfy $w_{\Lambda}=|\Lambda| w_{L}$ where $L=\operatorname{span} \Lambda$. We denote the natural action of $G$ on $\mathcal{E}_{d, n}$ arising from the $d$-th exterior power of the linear action on $\mathbb{R}^{n}$, by $(g, w) \mapsto g w$. Given a subspace $L \subset \mathbb{R}^{n}$ and a discrete subgroup $\Lambda$ we set

$$
A_{L} \stackrel{\text { def }}{=}\left\{a \in A: a w_{L}=w_{L}\right\} \text { and } A_{\Lambda} \stackrel{\text { def }}{=}\left\{a \in A: a w_{\Lambda}=w_{\Lambda}\right\} .
$$

Note that the requirement $a w_{L}=w_{L}$ is equivalent to saying that $a L=$ $L$ and $\operatorname{det}\left(\left.a\right|_{L}\right)=1$. Given a flag

$$
\mathscr{F}=\left\{0 \varsubsetneqq L_{1} \varsubsetneqq \cdots \varsubsetneqq L_{k} \varsubsetneqq \mathbb{R}^{n}\right\}
$$

(not necessarily full), let $A_{\mathscr{F}} \stackrel{\text { def }}{=} \bigcap_{i} A_{L_{i}}$. The support of an element $w \in \mathcal{E}_{d, n}$ is the subset of $\mathbf{I}_{d}^{n}$ for which the corresponding coefficients of an element of $\bigwedge^{d} \mathbb{R}^{n}$ representing $w$ are nonzero, and we write $\operatorname{supp}(L)$ or $\operatorname{supp}(\Lambda)$ for the supports of $w_{L}$ and $w_{\Lambda}$. For $J=\left\{i_{1}<\cdots<i_{d}\right\} \in \mathbf{I}_{d}^{n}$, set $\mathbb{R}^{J} \stackrel{\text { def }}{=} \operatorname{span}\left(e_{i_{j}}\right)$ and define the multiplicative characters

$$
\chi_{J}: A \rightarrow \mathbb{R}^{*}, \chi_{J}(a) \stackrel{\text { def }}{=} \operatorname{det}\left(\left.a\right|_{\mathbb{R}^{J}}\right) .
$$

Then for any subspace $L \subset \mathbb{R}^{n}$,

$$
A_{L}=\bigcap_{J \in \operatorname{supp}(L)} \operatorname{ker} \chi_{J}
$$

(and similarly for discrete subgroups $\Lambda$ ). As in $\S 3$ we fix an invariant metric on $A$. In order to verify hypothesis (ii) of Theorem 7.2, we will need the following lemmas (cf. [McM05, Theorem 6.1]):

Lemma 7.3. Let $T \subset A$ be a closed subgroup and let $x \in \mathcal{L}_{n}$ be a lattice with a compact $T$-orbit. Then for any $C>0$ there exists $R>0$ such that for any collection $\left\{\Lambda_{i}\right\}$ of subgroups of $x$, there exists $b \in A$ such that

$$
\left\{a \in T: \forall i\left\|a w_{\Lambda_{i}}\right\| \leq C\right\} \subset R \text {-neighborhood of } b\left(\bigcap_{i} A_{\Lambda_{i}}\right) .
$$

Proof. We will identify $A$ with its Lie algebra $\mathfrak{a}$ via the exponential map, and think of the subgroups $A_{\Lambda}$ as subspaces. By (7.2) only finitely many subspaces arise as $A_{\Lambda}$. In particular, given a collection of discrete 
subgroups $\left\{\Lambda_{i}\right\}$, the angles between the spaces they span (if nonzero) are bounded below. Therefore there exists a function $\psi: \mathbb{R} \rightarrow \mathbb{R}$ with $\psi(R) \rightarrow_{R \rightarrow \infty} \infty$, such that

$$
\begin{aligned}
& \left\{a \in A: \forall J \in \cup_{i} \operatorname{supp}\left(w_{\Lambda_{i}}\right), \psi(R)^{-1} \leq \chi_{J}(a) \leq \psi(R)\right\} \subset \\
& \left\{a \in A: d\left(a, \cap_{i} A_{\Lambda_{i}}\right) \leq R\right\} .
\end{aligned}
$$

Since $T x$ is compact, there exists a compact subset $\Omega \subset T$ such that for any $a \in T$ there exists $b=b(a) \in T$ satisfying $b x=x$ and $b^{-1} a \in \Omega$. It follows that there exists $M \geq 1$ such that:

(I) for any subspace $L,\left\|b w_{L}\right\| \leq M\left\|a w_{L}\right\|$.

(II) for any multi-index $J, \chi_{J}\left(b \bar{a}^{-1}\right) \leq M$.

Given $C>0$, let $C^{\prime} \stackrel{\text { def }}{=} M C$ and consider the finite set

$$
\mathscr{S} \stackrel{\text { def }}{=}\left\{\Lambda \subset x:\left\|w_{\Lambda}\right\| \leq C^{\prime}\right\}
$$

For any $\Lambda \in \mathscr{S}$ write $w_{\Lambda}=\sum_{J \in \operatorname{supp}\left(w_{\Lambda}\right)} \alpha_{J}(\Lambda) e_{J}$. Let $\varepsilon>0$ be small enough so that

$$
\varepsilon<\min \left\{\left|\alpha_{J}(\Lambda)\right|: \Lambda \in \mathscr{S}, J \in \operatorname{supp}\left(w_{\Lambda}\right)\right\}
$$

and choose $R$ large enough so that $\psi(R)>C^{\prime} / \varepsilon$. We claim that for any $\left\{\Lambda_{i}\right\} \subset \mathscr{S}$,

$$
\left\{a \in T: \forall i\left\|a w_{\Lambda_{i}}\right\| \leq C\right\} \subset\left\{a \in T: d\left(a, \cap_{i} A_{\Lambda_{i}}\right) \leq R\right\} .
$$

To prove this claim, suppose $a$ is an element on the left hand side of (7.5). By (7.4) it is enough show that for any $J \in \cup_{i} \operatorname{supp}\left(\Lambda_{i}\right)$ we have $\psi(R)^{-1} \leq \chi_{J}(a) \leq \psi(R)$. Since the coefficient of $e_{J}$ in the expansion of $a w_{\Lambda_{i}}$ is $\chi_{J}(a) \alpha_{J}\left(\Lambda_{i}\right)$ and since $\left\|a w_{\Lambda_{i}}\right\| \leq C$, we have

$$
\chi_{J}(a) \leq \frac{C}{\left|\alpha_{J}\left(\Lambda_{i}\right)\right|} \leq \frac{C}{\varepsilon} \leq \psi(R) .
$$

On the other hand, letting $b=b(a)$ we have $b \Lambda_{i} \in \mathscr{S}$ from (I), and

$$
\begin{aligned}
\varepsilon \leq\left|\alpha_{J}\left(b \Lambda_{i}\right)\right| & =\chi_{J}(b)\left|\alpha_{J}\left(\Lambda_{i}\right)\right| \Longrightarrow \chi_{J}\left(b^{-1}\right) \leq C / \varepsilon \\
& \stackrel{(\mathrm{II})}{\Longrightarrow} \chi_{J}\left(a^{-1}\right)=\chi_{J}\left(a^{-1} b\right) \chi_{J}\left(b^{-1}\right) \leq C^{\prime} / \varepsilon \leq \psi(R),
\end{aligned}
$$

which completes the proof of (7.5).

Let $\left\{\Lambda_{i}\right\}$ be any collection of subgroups of $x$ and assume that the set on the left hand side of (7.3) is non-empty. That is, there exists $a_{0} \in T$ such that for all $i,\left\|a_{0} w_{\Lambda_{i}}\right\| \leq C$. Let $b=b\left(a_{0}\right) \in T$, and set 
$\Lambda_{i}^{\prime} \stackrel{\text { def }}{=} b \Lambda_{i}$. It follows that $\left\{\Lambda_{i}^{\prime}\right\} \subset \mathscr{S}$ and so

$$
\begin{aligned}
\left\{a \in T: \forall i\left\|a w_{\Lambda_{i}}\right\| \leq C\right\} & =b\left\{a \in T: \forall i\left\|a w_{\Lambda_{i}^{\prime}}\right\| \leq C\right\} \\
& \stackrel{(7.5)}{\subset} b\left\{a \in T: d\left(a, \cap_{i} A_{\Lambda_{i}^{\prime}}\right) \leq R\right\} \\
& =\left\{a \in T: d\left(a, b\left(\cap_{i} A_{\Lambda_{i}}\right)\right) \leq R\right\},
\end{aligned}
$$

where in the last equality we used the fact that $A_{\Lambda_{i}^{\prime}}=A_{\Lambda_{i}}$ because $A$ is commutative.

Lemma 7.4. Let $\mathscr{F}$ be a flag as in (7.1) and let $A_{\mathscr{F}}$ be its stabilizer. Then $A_{\mathscr{F}}$ is of co-dimension $\geq k$ in $A$.

Proof. Given a nested sequence of multi-indices $J_{1} \varsubsetneqq \cdots \varsubsetneqq J_{k}$ it is clear that the subgroup

$$
\bigcap_{i=1}^{k} \operatorname{ker} \chi_{J_{i}}
$$

is of co-dimension $k$ in $A$. In light of (7.2), it suffices to prove the following claim:

Let $\mathscr{F}$ be a flag as in (7.1) with $d_{i} \stackrel{\text { def }}{=} \operatorname{dim} L_{i}$. Then there is a nested sequence of multi-indices $J_{i} \in \boldsymbol{I}_{d_{i}}^{n}$ such that $J_{i} \in \operatorname{supp}\left(L_{i}\right)$.

In proving the claim we will assume with no loss of generality that the flag is complete. Let $v_{1}, \ldots, v_{n}$ be a basis of $\mathbb{R}^{n}$ such that $L_{i}=$ $\operatorname{span}\left\{v_{j}\right\}_{j=1}^{i}$ for $i=1, \ldots, n-1$. Let $T$ be the $n \times n$ matrix whose columns are $v_{1}, \ldots, v_{n}$. Given a multi-index $J$ of length $|J|$, we denote by $T_{J}$ the square matrix of dimension $|J|$ obtained from $T$ by deleting the last $n-|J|$ columns and the rows corresponding to the indices not in $J$. Note that with this notation, possibly after replacing some of the $v_{i}$ 's by their scalar multiples, each $w_{L_{d}}$ is the image in $\mathcal{E}_{d, n}$ of

$$
v_{1} \wedge \cdots \wedge v_{d}=\sum_{J \in \mathbf{I}_{d}^{n}}\left(\operatorname{det} T_{J}\right) e_{J} .
$$

In particular, $J \in \operatorname{supp}\left(L_{d}\right)$ if and only if $\operatorname{det} T_{J} \neq 0$.

Proceeding inductively in reverse, we construct the nested sequence $J_{d}$ by induction on $d=n, \ldots, 1$. Let $J_{n}=\{1, \ldots, n\}$ so that $T=T_{J_{n}}$. Suppose we are given multi-indices $J_{n} \supset \cdots \supset J_{d+1}$ such that $J_{i} \in$ $\operatorname{supp}\left(w_{L_{i}}\right)$ for $i=n, \ldots, d+1$. We want to define now a multi index $J_{d} \in \operatorname{supp}\left(w_{L_{d}}\right)$ which is contained in $J_{d+1}$. By (7.6), $\operatorname{det} T_{J_{d+1}} \neq 0$. When computing $\operatorname{det} T_{J_{d+1}}$ by expanding the last column we express $\operatorname{det} T_{j_{d+1}}$ as a linear combination of $\left\{\operatorname{det} T_{J}: J \subset J_{d+1},|J|=d\right\}$. We conclude that there must exist at least one multi-index $J_{d} \subset J_{d+1}$ for 
which $\operatorname{det} T_{J_{d}} \neq 0$. In turn, by (7.6) this means that $J_{d} \in \operatorname{supp}\left(w_{L_{d}}\right)$. This finishes the proof of the claim.

The following notation is analogous to Definition 2.5. Given a lattice $x \in \mathcal{L}_{n}$ and $\delta>0$ let

$$
\begin{aligned}
\operatorname{Min}_{\delta}^{*}(x) & \stackrel{\text { def }}{=}\left\{v \in x \backslash\{0\}:\|v\|<(1+\delta) \alpha_{1}(x)\right\} . \\
\mathbf{V}_{\delta}^{*}(x) & \stackrel{\text { def }}{=} \operatorname{span} \operatorname{Min}_{\delta}^{*}(x) . \\
\operatorname{dim}_{\delta}^{*}(x) & \stackrel{\text { def }}{=} \operatorname{dim} \mathbf{V}_{\delta}^{*}(x) .
\end{aligned}
$$

Finally, for $\varepsilon>0$, let $\mathcal{U}^{(\varepsilon)}=\left\{U_{j}^{(\varepsilon)}\right\}_{j=1}^{n}$ be the collection of open subsets of $A$ defined by

$U_{j}=U_{j}^{(\varepsilon)} \stackrel{\text { def }}{=}\left\{a \in A\right.$ : for all $\delta$ in a neighborhood of $\left.j \varepsilon, \operatorname{dim}_{\delta}^{*}(a x)=j\right\}$.

Similarly to the discussion in Lemma 3.4 we see that $\mathcal{U}^{(\varepsilon)}$ is an open cover of $A$.

Proof of Theorem 7.1. The strategy of proof is very similar to that of Theorem 1.1. We consider covers $\mathcal{U}^{(\varepsilon)}$ of $A$ and use Theorem 7.2 to deduce that $U_{n}^{(\varepsilon)}$ is non-empty. The first step towards applying Theorem 7.2 is to find a decomposition $A \simeq \mathbb{R}^{n-1}=\mathbb{R}^{s} \times \mathbb{R}^{t}$ and a simplex $\Delta \subset \mathbb{R}^{s}$, so that the restriction of the cover to $\Delta \times \mathbb{R}^{t}$ satisfies the two hypotheses of Theorem 7.2.

According to [TW03, SW], there is a decomposition $A=T_{1} \times T_{2}$ and a direct sum decomposition $\mathbb{R}^{n}=\bigoplus_{1}^{d} V_{i}$ such that the following hold:

- Each $V_{i}$ is spanned by some of the standard basis vectors.

- $T_{1}$ is the group of linear transformations which act on each $V_{i}$ by a homothety, preserving Lebesgue measure on $\mathbb{R}^{n}$. In particular $s \stackrel{\text { def }}{=} \operatorname{dim} T_{1}=d-1$.

- $T_{2}$ is the group of diagonal (with respect to the standard basis) matrices whose restriction to each $V_{i}$ has determinant 1.

- $T_{2} x$ is compact and $T_{1} x$ is divergent; i.e. $A x \cong T_{1} \times T_{2} /\left(T_{2}\right)_{x}$, where $\left(T_{2}\right)_{x} \stackrel{\text { def }}{=}\left\{a \in T_{2}: a x=x\right\}$.

- Setting $\Lambda_{i} \stackrel{\text { def }}{=} V_{i} \cap x$, each $\Lambda_{i}$ is a lattice in $V_{i}$, so that $\bigoplus \Lambda_{i}$ is of finite index in $x$.

For $a \in T_{1}$ we write $\chi_{i}(a)$ for the number satisfying $a v=e^{\chi_{i}(a)} v$ for all $v \in V_{i}$. Thus each $\chi_{i}$ is a homomorphism from $T_{1}$ to the additive group of real numbers. The mapping $a \mapsto \bigoplus_{i} \chi_{i}(a) \operatorname{Id}_{V_{i}}$, where $\operatorname{Id}_{V_{i}}$ is the identity map on $V_{i}$, is nothing but the logarithmic map of $T_{1}$ and 
it endows $T_{1}$ with the structure of a vector space. In particular we can discuss the convex hull of subsets of $T_{1}$. For each $\rho$ we let

$$
\Delta_{\rho} \stackrel{\text { def }}{=}\left\{a \in T_{1}: \max _{i} \chi_{i}(a) \leq \rho\right\} .
$$

Then $\Delta_{\rho}=\operatorname{conv}\left(b_{1}, \ldots, b_{d}\right)$ where $b_{i}$ is the diagonal matrix acting on each $V_{j}, j \neq i$ by multiplication by $e^{\rho}$, and contracting $V_{i}$ by the appropriate constant ensuring that $\operatorname{det} b_{i}=1$.

Let $P_{i}: \mathbb{R}^{n} \rightarrow V_{i}$ be the natural projection associated with the decomposition $\mathbb{R}^{n}=\bigoplus V_{i}$. Each $P_{i}(x)$ is of finite index in $\Lambda_{i}$ and hence discrete in $V_{i}$. Moreover, the orbit $T_{2} x$ is compact, so for each $a \in T_{2}$ there is $a^{\prime}$ belonging to a bounded subset of $T_{2}$ such that $a x=a^{\prime} x$. This implies that there is $\eta>0$ such that for any $i$ and any $a \in T_{2}$, if $v \in a x$ and $P_{i}(v) \neq 0$ then $\left\|P_{i}(v)\right\| \geq \eta$. Let $C>0$ be large enough so that $\alpha_{1}\left(x^{\prime}\right) \leq C$ for any $x^{\prime} \in \mathcal{L}_{n}$. Let $\rho$ be large enough so that

$$
e^{\rho} \eta>2 C \text {. }
$$

We restrict the covers $\mathcal{U}^{(\varepsilon)}$ (where $\varepsilon \in(0,1 / n)$ ) to $\Delta_{\rho} \times T_{2}$ and apply Theorem 7.2 with $t \stackrel{\text { def }}{=} \operatorname{dim} T_{2}=n-d$. If we show that the hypotheses of Theorem 7.2 are satisfied for each cover $\mathcal{U}^{(\varepsilon)}$, we will obtain $U_{n}^{(\varepsilon)} \neq \varnothing$. Then, taking $\varepsilon_{j} \rightarrow 0$ and applying a compactness argument, we find a well-rounded lattice in $\left(\Delta_{\rho} \times T_{2}\right) x$.

Let $U$ be a connected subset of $U_{k}^{(\varepsilon)} \in \mathcal{U}^{(\varepsilon)}$. Repeating the arguments proving Lemma 3.5, or appealing to [McM05, §7], we see that the $k$ dimensional subspace $L \stackrel{\text { def }}{=} a^{-1} \mathbf{V}_{k \varepsilon}^{*}(a x)$ as well as the discrete subgroup $\Lambda \stackrel{\text { def }}{=} L \cap x$ are independent of the choice of $a \in U$. By definition of $U_{k}^{(\varepsilon)}$, for any $a \in U, a \Lambda$ contains $k$ vectors $v_{i}=v_{i}(a), i=1, \ldots, k$ which span $a L$ and satisfy

$$
\left\|v_{i}\right\| \in[r,(1+k \varepsilon) r], \text { where } r \stackrel{\text { def }}{=} \alpha_{1}(a x) .
$$

In order to verify hypothesis (i) of Theorem 7.2 , we need to show that there is at least one $j$ for which $U \cap M_{j}=\varnothing$. Let $P_{1}, \ldots, P_{d}$ be the projections above. Since ker $P_{1} \cap \cdots \cap \operatorname{ker} P_{d}=\{0\}$ and $\operatorname{dim} L=k \geq 1$, it suffices to show that whenever $U \cap M_{j} \neq \varnothing, L \subset$ ker $P_{j}$. The face $F_{j}$ of $\Delta_{\rho}$ consists of those elements $a_{1} \in T_{1}$ which expand vectors in $V_{j}$ by a factor of $e^{\rho}$. If $U \cap M_{j} \neq \varnothing$ then there is $a \in T_{2}, a_{1} \in F_{j}$ so that $a_{1} a \in U$. Now (7.8), (7.9) and the choice of $\eta$ and $C$ ensure that the vectors $v_{i}=v_{i}\left(a_{1} a\right)$ satisfy $P_{j}\left(v_{i}\right)=0$. Therefore $L \subset \operatorname{ker} P_{j}$.

It remains to verify hypothesis (ii) of Theorem 7.2. Let $U$ be a connected subset of an intersection $U_{i_{1}} \cap \cdots \cap U_{i_{k}} \cap\left(\Delta_{\rho} \times T_{2}\right)$ and let 
$L_{i_{j}} \stackrel{\text { def }}{=} a^{-1} \mathbf{V}_{i_{j} \varepsilon}^{*}(a x)$ and $\Lambda_{i_{j}} \stackrel{\text { def }}{=} L_{i_{j}} \cap x$. As remarked above, $L_{i_{j}}, \Lambda_{i_{j}}$ are independent of $a \in U$.

By the definition of the $L_{i_{j}}$ 's we have that $L_{i_{j}} \varsubsetneqq L_{i_{j+1}}$ and so they form a flag $\mathscr{F}$ as in (7.1). Lemma 7.4 applies and we deduce that

$$
A_{\mathscr{F}}=\cap_{j=1}^{k} A_{L_{i}} \text { is of co-dimension } \geq k \text { in } A \text {. }
$$

For each $a \in U$ and each $j$ let $\left\{v_{\ell}^{(j)}(a)\right\} \in a \Lambda_{i_{j}}$ be the vectors spanning $a L_{i_{j}}$ which satisfy (7.9). Let $u_{\ell}^{(j)}(a) \stackrel{\text { def }}{=} a^{-1} v_{\ell}^{(j)} \in \Lambda_{i_{j}}$. Observe that:

(a) $\operatorname{span}_{\mathbb{Z}}\left\{u_{\ell}^{(j)}(a)\right\}$ is of finite index in $\Lambda_{i_{j}}$ and in particular, $u_{1}^{(j)}(a) \wedge$ $\cdots \wedge u_{i_{j}}^{(j)}(a)$ is an integer multiple of $\pm w_{\Lambda_{i_{j}}}$. As a consequence $\left\|a w_{\Lambda_{i_{j}}}\right\| \leq\left\|v_{1}^{(j)}(a) \wedge \cdots \wedge v_{i_{j}}^{(j)}(a)\right\|$.

(b) Because of (7.9) we have that $\left\|v_{1}^{(j)}(a) \wedge \cdots \wedge v_{i_{j}}^{(j)}(a)\right\|<C$ for some constant depending on $n$ alone.

It follows from (a),(b) and Lemma 7.3 that there exist $R>0$ and an element $b \in T_{2}$ so that

$U \subset \Delta_{\rho} \times\left\{a \in T_{2}: \forall i_{j},\left\|a w_{\Lambda_{i_{j}}}\right\|<C\right\} \subset T_{1} \times\left\{a \in T_{2}: d\left(a, b A_{\mathscr{F}}\right) \leq R\right\}$.

By (7.10) we deduce that if $p_{2}: A \rightarrow T_{2}$ is the projection associated with the decomposition $A=T_{1} \times T_{2}$ then $p_{2}(U)$ is $\left(R^{\prime}, s+t-k\right)$-almost afine, where $R^{\prime}$ depends only on $R, \rho$. This concludes the proof.

\section{Appendix A. Proof of Theorem 7.2}

Below $X$ will denote a second countable metric space. We will use calligraphic letters like $\mathcal{U}$ for collections of sets. The symbol mesh $(\mathcal{A})$ will denote the supremum of the diameters of the sets in $\mathcal{A}$. The symbol $\operatorname{Leb}(\mathcal{A})$ will denote the Lebesgue number of a cover $\mathcal{A}$, i.e. the supremum of all numbers $r$ such that each ball of radius $r$ in $X$ is contained in some element of $\mathcal{A}$. The symbol $\operatorname{ord}(\mathcal{A})$ will denote the largest number of distinct elements of $\mathcal{A}$ with non-empty intersection.

Definition A.1. A collection $\left\{X_{j}\right\}_{j \in \mathscr{J}}$ of subsets of $X$ is said to be uniformly of asymptotic dimension $\leq n$ if for every $r>0$ there is $R>0$ such that for every $j \in \mathscr{J}$ there is an open cover $\mathcal{X}_{j}$ of $X_{j}$ such that

- $\operatorname{mesh}\left(\mathcal{X}_{j}\right) \leq R$.

- $\operatorname{Leb}\left(\mathcal{X}_{j}\right)>r$.

- $\operatorname{ord}\left(\mathcal{X}_{j}\right) \leq n+1$.

As an abbreviation we will sometimes write 'asdim' in place of 'asymptotic dimension'. 
Recall that a cover of $X$ is locally finite if every $x \in X$ has a neighborhood which intersects finitely many sets in the cover. We call the intersection of $k$ distinct elements of $\mathcal{A}$ a $k$-intersection, and denote the union of all $k$-intersections by $[\mathcal{A}]^{k}$. We will need the following two Propositions for the proof of Theorem 7.2. We first prove Theorem 7.2 assuming them and then turn to their proof.

Proposition A.2. Let $\mathcal{A}$ be a locally finite open cover of a space $X$ such that $\operatorname{ord}(\mathcal{A}) \leq m$ and the collection of components of the $k$ intersections of $\mathcal{A}, 1 \leq k \leq m$, is uniformly of asdim $\leq m-k$. Then $\mathcal{A}$ can be refined by a uniformly bounded open cover of order at most $m$.

Proposition A.3. Let $\Delta_{1}$ and $\Delta_{2}$ be simplices, $X=\Delta_{1} \times \Delta_{2}, p_{i}$ : $X \rightarrow \Delta_{i}$ the projections and $\mathcal{A}$ a finite open cover of $X$ such that for every $A \in \mathcal{A}$ and $i=1,2$ the set $p_{i}(A)$ does not meet at least one of the faces of $\Delta_{i}$. Then $\operatorname{ord}(\mathcal{A}) \geq \operatorname{dim} \Delta_{1}+\operatorname{dim} \Delta_{2}+1$.

Proof of Theorem 7.2. Let $m \stackrel{\text { def }}{=} \operatorname{dim} M=s+t$, and suppose by contradiction that $\operatorname{ord}(\mathcal{U}) \leq m$. Since every cover of $M$ has a locally finite refinement, there is no loss of generality in assuming that $\mathcal{U}$ is locally finite. Replacing $\mathcal{U}$ with the set of connected components of elements of $\mathcal{U}$, we may assume that all elements of $\mathcal{U}$ are connected. For any $r_{0}$, and any bounded set $Y$, the product space $Y \times \mathbb{R}^{d}$ can be covered by a cover of order $d+1$ and Lebesgue number greater than $r_{0}$. Hence our hypothesis (ii) implies that for each $k=1, \ldots, m$, the collection of connected components of intersections of $k$ distinct elements of $\mathcal{U}$ is uniformly of asymptotic dimension at most $m-k$. Therefore we can apply Proposition A.2 to assume that $\mathcal{U}$ is uniformly bounded and of order at most $m$. Take a sufficiently large $t$-dimensional simplex $\Delta_{1} \subset \mathbb{R}^{t}$ so that the projection of every set in $\mathcal{U}$ does not intersect at least one of the faces of $\Delta_{1}$. We obtain a contradiction to Proposition A.3.

For the proofs of Propositions A.2, A.3 we will need some auxiliary lemmas.

Lemma A.4. Let $\left\{G_{i}: i \in \mathcal{I}\right\}$ be a locally finite collection of open subsets of a metric space $X$, and let $Z$ be an open subset such that for each $i \neq j, G_{i} \cap G_{j} \subset Z$. Then there are disjoint open subsets $\boldsymbol{E}_{i}, i \in \mathscr{I}$, such that for any $i$

$$
G_{i} \backslash Z \subset \boldsymbol{E}_{i} \subset G_{i} .
$$

Proof. For each $G_{i}$ and $x \in G_{i} \cap \partial\left(G_{i} \backslash Z\right)$ set

$$
r_{x} \stackrel{\text { def }}{=} \frac{1}{3} \inf _{j \neq i} d\left(x, \partial\left(G_{j} \backslash Z\right)\right),
$$


where $d$ is the metric on $X$. The infimum in this definition is in fact a minimum since $\left\{G_{i}\right\}$ is locally finite. To see that it is positive, suppose if possible that $y_{\ell} \rightarrow x$ for a sequence $\left(y_{\ell}\right) \subset \partial\left(G_{j} \backslash Z\right)$. Then there are $\tilde{y}_{\ell} \in G_{j} \backslash Z$ with $d\left(y_{\ell}, \tilde{y}_{\ell}\right) \rightarrow 0$ so that $\tilde{y}_{\ell} \rightarrow x$. Since $G_{i}$ is open, for large enough $\ell$ we have $\tilde{y}_{\ell} \in G_{i}$, contradicting the assumption that $G_{i} \cap G_{j} \subset Z$. Now we set

$\mathbf{E}_{i} \stackrel{\text { def }}{=} \mathbf{E}^{\prime} \cup \mathbf{E}^{\prime \prime}$, where $\quad \mathbf{E}^{\prime} \stackrel{\text { def }}{=} G_{i} \backslash Z$ and $\mathbf{E}^{\prime \prime} \stackrel{\text { def }}{=} G_{i} \cap \bigcup_{x \in G_{i} \cap \partial\left(G_{i} \backslash Z\right)} B\left(x, r_{x}\right)$.

Clearly each $\mathbf{E}_{i}$ satisfies (A.1), and it is open since $\mathbf{E}^{\prime \prime}$ is open and covers the boundary points of $\mathbf{E}^{\prime}$. To show that the sets $\mathbf{E}_{i}$ are disjoint, suppose if possible that $z \in \mathbf{E}_{i} \cap \mathbf{E}_{j}$. Then there are $x \in G_{i}, y \in G_{j}$ such that $z \in B\left(x, r_{x}\right) \cap B\left(y, r_{y}\right)$. Supposing with no loss of generality that $r_{x} \geq r_{y}$ we find that

$$
d(x, y) \leq d(x, z)+d(z, y) \leq 2 r_{x} \leq \frac{2}{3} d\left(x, \partial\left(G_{j} \backslash Z\right)\right),
$$

which is impossible.

We denote the nerve of a cover $\mathcal{A}$ by $\operatorname{Nerve}(\mathcal{A})$, and consider it with the metric topology induced by barycentric coordinates. Given a partitition of unity subordinate to a cover $\mathcal{A}$ of $X$, there is a standard construction of a map $X \rightarrow \operatorname{Nerve}(\mathcal{A})$; such a map is called a canonical map.

Lemma A.5. Let a space $Y$ be the union of two open subsets $\mathbf{D}$ and $\mathbf{E}$, and let $\mathcal{D}$ and $\mathcal{E}$ be open covers of $\mathbf{D}$ and $\mathbf{E}$ respectively, with bounded mesh and ord, and such that if $C \subset \mathbf{D} \cap \mathbf{E}$ is a connected subset contained in an element of $\mathcal{D}$, then it is contained in an element of $\mathcal{E}$. Then, there is an open cover $\mathcal{Y}$ of $Y$ such that:

(1) The cover $\mathcal{Y}$ refines $\mathcal{D} \cup \mathcal{E}$.

(2) $\operatorname{mesh}(\mathcal{Y}) \leq \max (\operatorname{mesh}(\mathcal{D}), \operatorname{mesh}(\mathcal{E}))$.

(3) $\operatorname{ord}(\mathcal{Y}) \leq \max (\operatorname{ord}(\mathcal{D})+1$, ord $(\mathcal{E}))$.

Proof. Let $\operatorname{ord}(\mathcal{D})=n+1$, let $X \stackrel{\text { def }}{=} \operatorname{Nerve}(\mathcal{D})$, and let $\pi: \mathbf{D} \rightarrow X$ be a canonical map. Take an open cover of $\mathcal{X}$ of $X$ such that $\operatorname{ord}(\mathcal{X}) \leq n+1$ and $\pi^{-1}(\mathcal{X})$ refines $\mathcal{D}$. Let $f: Y \rightarrow[0,1]$ be a continuous map such that $\left.f\right|_{Y \backslash \mathbf{E}} \equiv 0$ and $\left.f\right|_{Y \backslash \mathbf{D}} \equiv 1$. Set $Y^{\prime} \stackrel{\text { def }}{=} f^{-1}\left(\left[\frac{1}{3}, \frac{2}{3}\right]\right)$ and

$$
g: Y^{\prime} \rightarrow Z \stackrel{\text { def }}{=} X \times\left[\frac{1}{2}, \frac{2}{3}\right], \quad g(y) \stackrel{\text { def }}{=}(\pi(y), f(y)) .
$$

Since $\operatorname{dim} Z \leq n+1$ there is an open cover $\mathcal{Z}$ of $Z$ such that $\operatorname{ord}(\mathcal{Z}) \leq$ $n+2$, the projection of $\mathcal{Z}$ to $X$ refines $\mathcal{X}$ and the projection of $\mathcal{Z}$ 
to $\left[\frac{1}{2}, \frac{2}{3}\right]$ is of mesh $<1 / 3$. Let $\mathcal{Y}^{\prime}$ denote the collection of connected components of sets $\left\{g^{-1}(W): W \in \mathcal{Z}\right\}$. By construction $\mathcal{Y}^{\prime}$ refines $\mathcal{D}$. Also, since the sets in $\mathcal{Y}^{\prime}$ are connected and contained in $\mathbf{D} \cap \mathbf{E}$ the assumption of the Lemma implies that $\mathcal{Y}^{\prime}$ also refines $\mathcal{E}$. Moreover $\operatorname{ord}\left(\mathcal{Y}^{\prime}\right) \leq n+2$ and no element of $\mathcal{Y}^{\prime}$ meets both $f^{-1}\left(\frac{1}{3}\right)$ and $f^{-1}\left(\frac{2}{3}\right)$. For every $\Omega \in \mathcal{Y}^{\prime}$ which intersects $f^{-1}\left(\frac{1}{3}\right)$, there is an element $D \in \mathcal{D}$ such that $\Omega \subset D$. We choose one such $D$ and say that $D$ marks $\Omega$. Similarly if $\Omega$ intersects $f^{-1}\left(\frac{2}{3}\right)$ there is $E \in \mathcal{E}$ so that $\Omega \subset E$, we choose one such $E$ and say that $E$ marks $\Omega$. We now modify elements of $\mathcal{D}$ and $\mathcal{E}$ : for each element $D \in \mathcal{D}$, define

$$
\tilde{D} \stackrel{\text { def }}{=}\left(D \cap f^{-1}([0,1 / 3))\right) \cup \bigcup_{D \text { marks } \Omega} \Omega .
$$

Similarly we modify elements of $\mathcal{E}$, defining

$$
\tilde{E} \stackrel{\text { def }}{=}\left(E \cap f^{-1}((2 / 3,1])\right) \cup \bigcup_{E \text { marks } \Omega} \Omega .
$$

We refer to $\tilde{D}, \tilde{E}$ as modified elements of $\mathcal{D}, \mathcal{E}$. Finally define $\mathcal{Y}$ as the collection of modified elements of $\mathcal{D}$ and $\mathcal{E}$ and the elements of $\mathcal{Y}^{\prime}$ which do not meet $f^{-1}\left(\frac{1}{3}\right)$ or $f^{-1}\left(\frac{2}{3}\right)$. It is easy to see that $\mathcal{X}$ has the required properties.

Lemma A.6. Let $Y$ be a metric space and let $\mathbf{D}, \mathbf{E}_{i}, i \in \mathscr{I}$ be open subsets which cover $Y$. Assume that the $\mathbf{E}_{i}$ 's are disjoint, connected, and are uniformly of asdim $\leq \ell-1$. Let $\mathcal{D}$ be an open cover of $\mathbf{D}$ which is of bounded mesh and ord $\mathcal{D} \leq \ell$. Then $Y$ has an open cover $\mathcal{Y}$ which refines the cover $\mathcal{D} \cup\left\{\mathbf{E}_{i}: i \in \mathscr{I}\right\}$, is of bounded mesh and ord $\mathcal{Y} \leq \ell+1$.

Proof. Using the assumption that $\mathbf{E}_{i}$ is uniformly of asdim $\leq \ell-1$ we find an open cover $\mathcal{E}_{i}$ of $\mathbf{E}_{i}$ which is of uniformly bounded mesh, such that ord $\mathcal{E}_{i} \leq \ell$ and Leb $\mathcal{E}_{i}>$ mesh $\mathcal{D}$. Let $\mathbf{E} \stackrel{\text { def }}{=} \bigcup_{\mathscr{I}} \mathbf{E}_{i}$ and let

$$
\mathcal{E} \stackrel{\text { def }}{=} \bigcup_{i \in \mathscr{I}}\left\{\mathbf{E}_{i} \cap U: U \in \mathcal{E}_{i}\right\}
$$

Clearly it suffices to verify that the hypotheses of Lemma A.5 are satisfied. Indeed, by assumption the cover $\mathcal{D}$ is of bounded mesh and order, and $\mathcal{E}$ is of bounded mesh because of the uniform bound on $\operatorname{mesh}\left(\mathcal{E}_{i}\right)$. We also have that ord $\mathcal{E} \leq \ell+1$ because of the bounds $\operatorname{ord}_{i} \leq \ell+1$ and the fact that the $\mathbf{E}_{i}$ are disjoint. For the last condition, let a connected subset $C \subset \mathbf{D} \cap \mathbf{E}$ which is contained in an element of $\mathcal{D}$ be given. By the connectedness and disjointness of the $\mathbf{E}_{i}$ 's we conclude that there exists $i$ with $C \subset \mathbf{E}_{i}$. Because Leb $\mathcal{E}_{i}>$ 
mesh $\mathcal{D}$ we deduce that since $C$ is contained in an element of $\mathcal{D}$ it must be contained in an element of $\mathcal{E}_{i}$ and in turn, as it is contained in $\mathbf{E}_{i}$, it must be contained in an element of $\mathcal{E}$.

Proof of Proposition A.2. Proceeding inductively in reverse order, for $k=m, \ldots, 1$ we will construct a uniformly bounded open cover $\mathcal{A}^{k}$ of $[\mathcal{A}]^{k}$ such that ord $\left(\mathcal{A}^{k}\right) \leq m+1-k$ and $\mathcal{A}^{k}$ refines the restriction of $\mathcal{A}$ to $[\mathcal{A}]^{k}$. The construction is obvious for $k=m$. Namely, our hypothesis and Definition A.1 with $n=m-k=0$ mean that $[\mathcal{A}]^{m}$ has a cover of bounded mesh and order 1 , that is, we can just set $\mathcal{A}^{m}$ to be the connected components of $[\mathcal{A}]^{m}$. Assume that the construction is completed for $k+1$ and proceed to $k$ as follows. First notice that for two distinct $k$-intersections $A$ and $A^{\prime}$ of $\mathcal{A}$ the complements $A \backslash[\mathcal{A}]^{k+1}$ and $A^{\prime} \backslash[\mathcal{A}]^{k+1}$ are disjoint. By Lemma A.4, we can cover $[\mathcal{A}]^{k} \backslash[\mathcal{A}]^{k+1}$ by a collection $\left\{\mathbf{E}_{i}: i \in \mathscr{I}\right\}$ of disjoint connected open sets such that every $\mathbf{E}_{i}$ is contained in a $k$-intersection of $\mathcal{A}$. In particular, the collection $\left\{\mathbf{E}_{i}: i \in \mathscr{I}\right\}$ is uniformly of asdim $\leq m-k$. We can therefore apply Lemma A.6 with the choices $Y=[\mathcal{A}]^{k}, \mathbf{D}=[\mathcal{A}]^{k+1}, \mathcal{D}=\mathcal{A}^{k+1}$, the collection $\left\{\mathbf{E}_{i}: i \in \mathscr{I}\right\}$, and $\ell=m-k$, and obtain an open cover $\mathcal{Y}$ of $[\mathcal{A}]^{k}$ of order $\leq m-k+1$ that refines $\mathcal{D} \cup\left\{\mathbf{E}_{i}: i \in \mathscr{I}\right\}$ and in particular, refines $\left.\mathcal{A}\right|_{Y}$. This completes the inductive step.

Proofs of Proposition $A .3$. For every $A \in \mathcal{A}$ choose a vertex $v_{i}^{A}$ of $\Delta_{i}$ so that $p_{i}(A)$ does not intersect the face of $\Delta_{i}$ opposite to $v_{i}^{A}$. Let $Y \stackrel{\text { def }}{=} \operatorname{Nerve}(\mathcal{A})$ and let $f: X \rightarrow X$ be the composition of a canonical map $X \rightarrow Y$ and a map $Y \rightarrow X$ which is linear on each simplex of $Y$ and sends the vertex of $Y$ related to $A \in \mathcal{A}$ to the point $\left(v_{1}^{A}, v_{2}^{A}\right) \in X$. Take a point $x \in \partial \Delta_{1} \times \Delta_{2}$. Then $p_{1}(x)$ belongs to a face $\Delta_{1}^{\prime}$ of $\Delta_{1}$ and hence for every $A \in \mathcal{A}$ containing $x$ we have that $v_{1}^{A} \in \Delta_{1}^{\prime}$. Thus both $x$ and $f(x)$ belong to $\Delta_{1}^{\prime} \times \Delta_{2}$. Applying the same argument to $\Delta_{1} \times \partial \Delta_{2}$ we get that the boundary $\partial X$ is invariant under $f$ and $f$ restricted to $\partial X$ is homotopic to the identity map of $\partial X$. If $\operatorname{ord}(\mathcal{A}) \leq$ $\operatorname{dim} \Delta_{1}+\operatorname{dim} \Delta_{2}$ then $\operatorname{dim} Y \leq \operatorname{dim} X-1$ and hence there is an interior point $a$ of $X$ not covered by $f(X)$. Take a retraction $r: X \backslash\{a\} \rightarrow \partial X$. Then the identity map of $\partial X$ factors up to homotopy through the contractible space $X$ which contradicts the non-triviality of the reduced homology of $\partial X$. 


\section{REFERENCES}

[BC72] J. Brenner and L. Cummings, The Hadamard maximum determinant problem, Amer. Math. Monthly 79 (1972), 626-630. MR0301030 (46 \#190)

[BSD56] B. J. Birch and H. P. F. Swinnerton-Dyer, On the inhomogeneous minimum of the product of $n$ linear forms, Mathematika 3 (1956), 25-39. MR0079049 (18,22a)

[DS13] M. Dutour-Sikirić, Enumeration of inhomogeneous perfect forms, 2013. Manuscript in preparation.

[DSSV12] M. Dutour Sikirić, A. Schürmann, and F. Vallentin, Inhomogeneous extreme forms, Ann. Inst. Fourier (Grenoble) (2012).

[Gra84] D. R. Grayson, Reduction theory using semistability, Comment. Math. Helv. 59 (1984), no. 4, 600-634. MR780079 (86h:22018)

[HGRS09] R. J. Hans-Gill, M. Raka, and R. Sehmi, On conjectures of Minkowski and Woods for $n=7$, J. Number Theory 129 (2009), no. 5, 1011-1033. MR2516969 (2010i:11099)

[HGRS11] R. J. Hans-Gill, M. Raka, and R. Sehmi, On conjectures of Minkowski and Woods for $n=8$, Acta Arith. 147 (2011), no. 4, 337-385. MR2776094 (2012b:11106)

[HN74] G. Harder and M. S. Narasimhan, On the cohomology groups of moduli spaces of vector bundles on curves, Math. Ann. 212 (1974/75), 215-248. MR0364254 (51 \#509)

[McM05] C. T. McMullen, Minkowski's conjecture, well-rounded lattices and topological dimension, J. Amer. Math. Soc. 18 (2005), no. 3, 711-734 (electronic). MR2138142 (2006a:11086)

[PS] A. Pettet and J. Suoto, Periodic maximal flats are not peripheral. preprint.

[Rag72] M. S. Raghunathan, Discrete subgroups of Lie groups, Springer-Verlag, New York, 1972. Ergebnisse der Mathematik und ihrer Grenzgebiete, Band 68. MR0507234 (58 \#22394a)

[Ram00] G. Ramharter, On the densities of certain lattice packings by parallelepipeds, Acta Math. Hungar. 88 (2000), no. 4, 331-340. MR1789045 (2001k:11122)

[Ram96] G. Ramharter, On Mordell's inverse problem in dimension three, J. Number Theory 58 (1996), no. 2, 388-415. MR1393622 (97h:11070)

[RL] M. Raka and Leetika, in prepration.

[Sie45] C. L. Siegel, A mean value theorem in geometry of numbers, Ann. of Math. (2) 46 (1945), 340-347. MR0012093 (6,257b)

[Stu76] U. Stuhler, Eine Bemerkung zur Reduktionstheorie quadratischer Formen, Arch. Math. (Basel) 27 (1976), no. 6, 604-610. MR0424707 (54 \#12666)

[SW] U. Shapira and B. Weiss, On the mordell-gruber spectrum. Preprint, available on arXiv at arxiv.org/pdf/1207.6343.

[Thu98] J. L. Thunder, Higher-dimensional analogs of hermite's constant., The Michigan Mathematical Journal 45 (1998), no. 2, 301-314.

[TW03] G. Tomanov and B. Weiss, Closed orbits for actions of maximal tori on homogeneous spaces, Duke Math. J. 119 (2003), no. 2, 367-392. MR1997950 (2004g:22006) 
[Wei82] A. Weil, Adeles and algebraic groups, Progress in Mathematics, vol. 23, Birkhäuser Boston, Mass., 1982. With appendices by M. Demazure and Takashi Ono. MR670072 (83m:10032)

[Wik] Wikipedia, Hadamard determinant problem.

[Woo65] A. C. Woods, The densest double lattice packing of four-spheres, Mathematika 12 (1965), 138-142. MR0193067 (33 \#1289a)

Dept. of Mathematics, Technion, Haifa, Israel put email here

Dept. of Mathematics, Tel Aviv University, Tel Aviv, Israel barakw@post.tau.ac.il 